A N N A L E S Annales de Bretagne et des Pays de l'Ouest

Anjou. Maine. Poitou-Charente. Touraine

114-1 | 2007

Varia

\title{
Étude des Vies de saint Ursin de Bourges : une première approche
}

\section{Nathalie Le Luel}

\section{(C) OpenEdition}

1 Journals

\section{Édition électronique}

URL : http://journals.openedition.org/abpo/589

DOI : $10.4000 / a b p o .589$

ISBN : 978-2-7535-1505-5

ISSN : 2108-6443

Éditeur

Presses universitaires de Rennes

Édition imprimée

Date de publication : 30 mars 2007

Pagination : 7-32

ISBN : 978-2-7535-0452-3

ISSN : 0399-0826

\section{Référence électronique}

Nathalie Le Luel, «Étude des Vies de saint Ursin de Bourges : une première approche », Annales de Bretagne et des Pays de l'Ouest [En ligne], 114-1 | 2007, mis en ligne le 30 mars 2009, consulté le 19 avril 2019. URL : http://journals.openedition.org/abpo/589 ; DOI : 10.4000/abpo.589 


\title{
Étude des Vies de saint Ursin de Bourges : Une première approche ${ }^{1}$
}

\author{
Nathalie LE LUEL \\ Doctorante en histoire de l'art, \\ Université Rennes 2 Haute-Bretagne
}

Au Xle siècle, l'Église de Bourges, à l'image de nombreux sièges épiscopaux contemporains, chercha à remettre en valeur les premiers temps de sa christianisation ${ }^{2}$, tentant de prouver le passé apostolique de celui que la tradition hagiographique considérait comme le premier évêque de la ville, Ursin ${ }^{3}$. Grand évangélisateur de la cité berruyère, saint Ursin aurait également favorisé la christianisation dans l'ensemble du Berry pour, à sa mort, laisser derrière lui une Église pleinement constituée.

La légende commence à vivre sous forme écrite à partir de la fin du $\mathrm{Vl}^{\mathrm{e}}$ siècle dans deux passages rédigés par l'évêque Grégoire de Tours et

1. Cet article est tiré, en grande partie, du premier chapitre du mémoire de Maîtrise d'Histoire du Moyen Âge, réalisé en 2000-2001, sous la direction de Bernard Merdrignac à l'Université Rennes 2 et ayant pour titre : La légende de fondation de l'église Saint-Ursin de Bourges : histoire et hagiographie. Je tiens à exprimer toute ma gratitude à mon directeur de recherche pour avoir dirigé ce travail de maîtrise et accepté de relire cet article à son terme. Que soient aussi remerciés ici Corinna Bottiglieri et Silvia Toniato pour leur aide amicale.

2. Devailly, Guy (dir.), Le Diocèse de Bourges, (Histoire des diocèses de France), Paris, 1973, p. 7 : "On pensait en effet aux XII ${ }^{\mathrm{e}}$ et XIII ${ }^{\mathrm{e}}$ siècles que l'antériorité d'un siège épiscopal donnait à son titulaire une sorte de prééminence sur les évêques voisins. Bourges qui réclamait non seulement la direction d'une province ecclésiastique mais la primatie sur l'ensemble de l'Aquitaine et parfois même sur toute la moitié méridionale de la France se devait de revendiquer une très haute antiquité. C'est l'époque où on prétendit identifier le premier évêque de Bourges saint Ursin à un disciple du Christ mentionné dans l'Évangile, Nathanaël ». La réflexion menée ici par Guy Devailly, même s'il évoque seulement les $\mathrm{XII}^{\mathrm{e}}$-XIII ${ }^{\mathrm{e}}$ siècles, s'applique, à l'aune des recherches actuelles, dès le $\mathrm{XI}^{\mathrm{e}}$ siècle.

3. En 1689, date de la première parution de l'ouvrage de Gaspard Thaumas de la Thaumassière, l'historien moderne écrivait : " Tous les auteurs conviennent que saint Ursin est l'Apôtre de Bourges, le premier de nos prélats, celuy qui aprez avoir banni les Prêtres des Idoles, ôté leurs Sacrifices, ruiné leurs Temples, extirpé l'Idolâtrie, y sema par ses prédictions la Doctrine de l'Évangile, y arbora l'Étendart de la Croix et établit le christianisme..." : ThAUMAS DE LA THAUMASsiÈRE, Gaspard, Histoire de Berry et du diocèse de Bourges, vol. 2, Bourges, 1865, p. 132. 
issus, pour l'un, de son œuvre majeure, l'Historia Francorum ${ }^{4}$ (chapitre XxI), pour l'autre, du Liber in Gloria Confessorum ${ }^{5}$ (chapitre LXXIx). Ces deux textes de Grégoire de Tours forment le premier fonds permettant de connaître l'hagiographie du saint berrichon. La seconde source, plus tardive, est celle sur laquelle nous concentrerons notre attention : elle est constituée du corpus de Vitae conservées d'Ursin. La rédaction quasi-simultanée des deux Vies du saint s'insère parfaitement dans le contexte du mouvement historique de quête des origines apostoliques ${ }^{6}$.

Les Vitae d'Ursin nous apprennent que le premier évêque de Bourges fut envoyé depuis Rome pour évangéliser le Berry. Peu avant l'arrivée au but final de son voyage, il perd son compagnon de route, Just. Chassé une première fois de Bourges, il regagne la ville et y poursuit son œuvre d'évangélisation. Il établit sa première église dans les écuries du sénateur des Gaules, Leocadius, mais, les foules affluant, celle-ci s'avère rapidement trop exiguë. Le sénateur, sensible aux prières d'Ursin, finit par lui céder le palais qu'il possédait dans la ville, où sont alors déposées les reliques du martyr Étienne, et se convertit au christianisme tout comme l'un de ses fils, Ludre. Après 27 ans d'épiscopat, Ursin, prévenu qu'il est sur le point d'être rappelé par le Seigneur, choisit son successeur, Sénécien, et meurt.

\section{Un corpus diversifié}

Le corpus hagiographique de saint Ursin (Ursinus), d'après le recensement effectué par les Bollandistes dans la Bibliotheca Hagiographica Latina ${ }^{7}$, se présente ainsi : deux Vitae différentes ont été dénombrées (BHL 8412 et 8413). On possède également un récit de revelatio (BHL 8411) qui n'est autre que le chapitre LXXIX du Liber in Gloria Confessorum écrit par Grégoire de Tours et enfin trois epitomae, récits abrégés de la vie de l'évêque de Bourges (BHL 8414, 8415 et 8415b).

La Vita BHL 8412, qu'on désignera par la lettre $\mathrm{A}^{8}$, se compose de 9 leçons sans prologue. Elle a été publiée deux fois au xixe siècle, la pre-

4. "Gregorii episcopi Turonensis Historiarum Liber I", dans Monumenta Germaniae Historica, éd. KRUSCH, Bernard, Scriptorum rerum merovingicarum, Tomus I Pars I, Fasc. I, Gregorii episcopi Turonensis Historiarum Libri X, Hanovre, 1885, p. 22-24.

5. "Gregorii episcopi Turonensis Liber in Gloria Confessorum ", dans Monumenta Germaniae Historica, éd. Krusch, Bernard, Scriptorum rerum merovingicarum, Tomus I Pars II, Gregorii episcopi Turonensis Miracula et opera minora, Hanovre, 1885, p. 346-348.

6. L'abbé ViLLEPELET écrivait, en 1931, à propos des Acta sancti Ursini : "Ils s'apparentent à cet ensemble de légendes écrites à une époque tardive, dans l'intention de faire remonter aux temps apostoliques les origines de telle ou telle église des Gaules " (Nos saints berrichons, Bourges, 1931, p. 192).

7. Bibliotheca Hagiographica Latina Antiquae et Mediae Aetatis, vol. 2 (K-Z), Bruxelles, 1900-1901, p. 1216. L'épitomé BHL 8415b n'est référencé qu'à partir de la Bibliotheca Hagiographica Latina antiquae et mediae aetatis, Novum supplementum, Bruxelles, 1986, p. 847. Voir également le site en ligne de la Bibliotheca Hagiographica Latina Manuscripta (index analytique des catalogues de manuscrits hagiographiques latins publiés par les Bollandistes : http://bhlms.fltr.ucl.ac.be/presentation.cfm).

8. Nous reprenons la désignation déjà en cours dans les Acta Santorum concernant 
mière fois par le prêtre sulpicien, Étienne-Michel Faillon, dans le deuxième volume des Monuments inédits sur l'apostolat de Sainte-Marie-Madeleine, en Provence et sur les autres apôtres de cette contrée ${ }^{9}$ sous le titre d'Acta sancti Ursini et la seconde par l'abbé C. Narbey ${ }^{10}$. Les deux auteurs ont puisé le texte latin dans un manuscrit parisien conservé aujourd'hui à la Bibliothèque Nationale de France sous la cote ms. Lat. 13220, aux folios 13 à 19. Daté des $\mathrm{X}^{\mathrm{e}}$-xl ${ }^{\mathrm{e}}$ siècles ${ }^{11}$, le manuscrit est un lectionnaire et antiphonaire du monastère Saint-Martial de Limoges, ayant appartenu au XVII ${ }^{\mathrm{e}}$ siècle à François de Harlay, archevêque de Rouen (1615-1652). Il fut ensuite légué par Achille IV de Harlay en 1716 à l'abbaye Saint-Germain-des-Prés, avant de faire partie des collections de la Bibliothèque Royale (ancienne cote Saint-Germ., Harlay, 369) ${ }^{12}$. Cependant la Vita A d'Ursin n'est pas conservée dans ce seul codex. On la retrouve dans d'autres manuscrits plus tardifs : un manuscrit berlinois daté du XI ${ }^{\mathrm{e}}$ siècle (Berlin, ms. 120, Phillips 1683, $\mathrm{f}^{\circ} 82-85 \mathrm{v}^{\circ}$ ), ainsi qu'un manuscrit de la Bibliothèque municipale d'Orléans (ms. 334, f ${ }^{\circ}$ 109-122) ${ }^{13}$. Ayant appartenu à l'abbaye de Saint-Benoît-sur-Loire, il est daté des $\mathrm{XI}^{\mathrm{e}}$-XII ${ }^{\mathrm{e}}$ siècles ${ }^{14}$. Deux autres manuscrits présentant la Vita A ont été inventoriés postérieurement par les Bollandistes. Ils sont conservés à la Bibliothèque municipale de Bourges. Il s'agit, pour le premier, du

les deux vies de saint Ursin : Delehaye, Hyppolite, Peeters, Paul, (éd.), Acta Santorum, Novembris, Tomus IV, Bruxelles, 1925, p. 101-115.

9. FAllLon, Étienne-Michel, Monuments inédits sur l'apostolat de Sainte-Marie-Madeleine, en Provence et sur les autres apôtres de cette contrée, vol. 2, Paris, 1848, col. 423-428. L'ouvrage appartient à ce courant de pensée, ayant fleuri au XIX ${ }^{\mathrm{e}}$ siècle, qui soutenait la thèse d'une origine apostolique du diocèse de Bourges, thèse sans doute remise à l'honneur par un auteur comme Lenain de Tillemont (Mémoires pour servir à l'histoire ecclésiastique des six premiers siècles, tome Iv, $3^{\text {e }}$ partie, Bruxelles, 1706, p. 1046-1047) au début du XVIII ${ }^{\mathrm{e}}$ siècle et reprise ensuite dans le Bréviaire de Bourges de 1734 (Pars autumn., aux 9 et 11 novembre). E. M. Faillon prônait en effet qu'Ursin se serait rendu à Bourges au $\mathrm{I}^{\mathrm{er}}$ siècle envoyé par saint Pierre.

10. NARBEY, Abbé C., Supplément aux Acta Sanctorum pour des Vies de Saints de l'Époque Mérovingienne, tome I, contenant des documents nouveaux ou peu connus sur toutes les églises des Gaules (50), qui se glorifient de remonter aux temps apostoliques ou quasi-apostoliques, Paris, 1899.

11. Il y a eu un important débat entre les auteurs à propos de ce manuscrit : les uns affirmant qu'il datait du Xe siècle (E. M. FAILLON, H. DELEHAYE), les autres du XI siècle (L. DUCHESNE, C. CHEVALIER). Léopold Delisle ne tranche pas et attribue le manuscrit aux Xe $-\mathrm{XI}^{\mathrm{e}}$ siècles : DeLISLE, Léopold, Inventaire des manuscrits latins, conservés à la Bibliothèque Nationale sous les numéros 8823-18613, vol. 1, Paris, 1863-1871, p. 94.

12. DelisLe, Léopold, Le Cabinet des manuscrits de la Bibliothèque nationale, t. II, Paris, 1874, p. 101 et 495; Catalogus codicum hagiographicorum latinorum antiquiorum saeculo XVI qui asservantur in Bibliotheca nationali Parisiensi, tomus III, Bruxelles/Paris, 1893, p. 183; SAMARAN, Charles et MARICHAL, Robert, Catalogue des manuscrits en écriture latine, portant des indications de date, de lieu ou de copiste, t. III, Paris, 1974, p. 319.

13. Recensement effectué par les Bollandistes et en particulier ici par le Père H. Delehaye : Delehaye, Hyppolite, « De sancto Ursino, primo biturigensi episcopo ", dans Delehaye, Hyppolite, PeETERS, Paul, (éd.), op. cit., p. 102.

14. Ce manuscrit est également mentionné dans l'étude de VAN DER STRAETEN, Joseph, Les manuscrits hagiographiques d'Orléans, Tours et Angers avec plusieurs textes inédits, Bruxelles, 1982, p. 62-63. 
manuscrit $\mathrm{n}^{\circ} 31$, un lectionnaire de 216 folios de parchemin réalisé au XII siècle et provenant de l'abbaye bénédictine de Chezal-Benoît dont les folios 5 verso et 6 présentent la Vita $\mathrm{A}^{15}$. Le second manuscrit ( $\left.\mathrm{n}^{\circ} 28\right)$, est aussi un lectionnaire de 352 folios achevé vers 1470; de provenance identique, il a d'ailleurs été copié sur le premier, le manuscrit $n^{\circ} 31^{16}$.

La seconde Vita de saint Ursin (BHL 8413), qu'on désignera par la lettre $\mathrm{B}$, possède un prologue suivi de 9 leçons ${ }^{17}$. Elle a été publiée par les Bollandistes eux-mêmes dans les Acta Sanctorum en 1925. Ils furent les premiers à éditer cette vie en son entier et à en proposer une version s'appuyant sur quatre manuscrits recensés par leurs soins : un manuscrit londonien daté du XII ${ }^{\mathrm{e}}$ siècle (Londres, British Museum, Royal 13. B. XIII, $\mathrm{f}^{\circ}$ 125-127), un manuscrit d'Oxford du XII ${ }^{\mathrm{e}}$ siècle (Oxford, Bodleian library, Fell $\left.3, f^{\circ} 77-81\right)$, un manuscrit qui appartient à la bibliothèque du chapitre de Bayeux (ms. 55, $\mathrm{f}^{\circ}$ 67-74) des $\mathrm{XII}^{\mathrm{e}}$-XIII ${ }^{\mathrm{e}}$ siècles et enfin un manuscrit parisien (Paris, BNF, ms. Lat. 14364, $\mathrm{f}^{\circ}$ 147-150) daté du XIII ${ }^{\mathrm{e}}$ siècle ${ }^{18}$. Ce dernier manuscrit ${ }^{19}$ est celui dans lequel Philippe Labbe a puisé la vie de saint Ursin imprimée dans l'ouvrage intitulé Novae Bibliothecae Manuscriptorum Librorum et publié en 1657 en l'honneur de Nicolas Fouquet ${ }^{20}$. La Vita B de saint Ursin est également conservée dans un manuscrit de la Bibliothèque municipale de Bourges, le manuscrit $\mathrm{n}^{\circ} 35$. Il s'agit du $2^{\mathrm{e}}$ tome d'un légendier latin en quatre volumes datant du début du $x v^{e}$ siècle. Ouvrage luxueux orné de miniatures, il fut probablement réalisé pour le chapitre de la SainteChapelle de Bourges, édifice aujourd'hui disparu, commandé par le duc Jean de Berry (1340-1416) ${ }^{21}$. Le numéro 35 se présente donc sous la forme d'un lectionnaire de 221 folios, les folios $202 \mathrm{v}^{\circ}$ à 207 proposant un texte qui correspond à une version abrégée de la Vita B d'Ursin.

Naturellement, le nombre des manuscrits recensés par les Bollandistes pourrait être encore accru par de nouvelles découvertes. Notre recherche sur le corpus hagiographique de saint Ursin atteste néanmoins de l'exis-

15. VAN DER STRAETEN, Joseph, " Manuscrits hagiographiques de Bourges ", dans Analecta Bollandiana, LXXXV, 1967, p. 75-112 et plus spécialement p. 89.

16. Ibidem, p. 77, 85-86 : La Vita d'Ursin occupe les folios 11-12 verso dans le manuscrit $\mathrm{n}^{\circ} 28$.

17. Bien que le découpage en leçons nous donne, aussi bien pour la Vita A que pour la Vita B, un total de 9, on s'aperçoit au premier coup d'œil de la longueur considérable des paragraphes de la $\mathrm{B}$ par rapport à ceux de la $\mathrm{A}$.

18. À la consultation des folios, il apparaît que les trois derniers paragraphes qui constituent la fin de la Vie de saint Ursin publiée par les Bollandistes manquent. Sur ce manuscrit, DELISLE, Léopold, Inventaire... op. cit., vol. 1, Paris, 1863-1871, p. 8 et 9.

19. Delehaye, Hyppolite, "De sancto Ursino, primo biturigensi episcopo ", dans op. cit., p. 102. On pourra également se référer pour ce manuscrit au Catalogus codicum hagiographicorum ..., op. cit., p. 228-233.

20. Cette Vie est également tronquée des trois paragraphes finaux de l'édition bollandiste et présente quelques variantes textuelles : LABBE, Philippe, Novae Bibliothecae Manuscriptorum Librorum, Tomus secundus, Rerum Aquitanicarum praesertim Bituricensium uberrima collectio, Paris, 1657, p. 455-459.

21. VAN DER StRAETEN, Joseph, "Manuscrits hagiographiques de Bourges ", dans op. cit., p. $77,79-84$. 
tence d'autres manuscrits recèlant une vie du premier évêque de Bourges sans qu'il soit précisé, dans les ouvrages ou articles qui les signalent, à quelle vie correspond le texte qui y est conservé. Le Catalogue général des manuscrits des bibliothèques publiques de France fait ainsi mention d'un lectionnaire $\left(\mathrm{n}^{\circ} 348\right.$ [288]) du XvI ${ }^{\mathrm{e}}$ siècle, conservé à la Bibliothèque municipale de Bourges, qui contient plusieurs vies d'évêques du diocèse dont une de saint Ursin ${ }^{22}$. Malheureusement, il n'est pas indiqué à quelle Vita celle-ci correspond et le manuscrit est aujourd'hui manquant à l'inventaire de la bibliothèque berruyère ${ }^{23}$. D'une manière identique, dans la revue Analecta Bollandiana, P. Grosjean citait en 1929 un manuscrit écossais (Edimbourg, Bibliothèque Nationale, NL, Codex 18.2.3.), daté du Xve siècle, dans lequel le folio 219 comprend une Vita d'Ursin placée dans un ensemble de vies consacrées à des disciples du Christ et des apôtres ${ }^{24}$. Appelé «BHL Ursinus " sur la base multimédia des Bollandistes, on ne possède aucun autre renseignement sur ce texte. Cependant si cette Vita " écossaise " n'a pas été classée sous les numéros déjà existants de la BHL, on peut imaginer que son texte est différent. On ne peut que regretter qu'il n'ait pas été publié.

Par ailleurs, notons que, dans les manuscrits précédemment cités, le texte d'une des deux Vitae est parfois compilé avec celui de la revelatio (BHL 8411), texte à l'origine indépendant ${ }^{25}$, écrit par Grégoire de Tours à la fin du vie siècle ${ }^{26}$. C'est le cas dans le manuscrit londonien conservé au British Museum (Royal 13. B. XIII, $\mathrm{f}^{\circ} 127 \mathrm{v}^{\circ}$ ), mais aussi dans le codex berlinois (ms. 120 , Phillips $\left.1683, \mathrm{f}^{\circ} 85 \mathrm{v}^{\circ}-87\right)^{27}$ et enfin dans le manuscrit de la Bibliothèque municipale d'Orléans (ms 334 [283]) aux folios 124-128 ${ }^{28}$. Il est vrai que ce chapitre LXXIX du Liber in Gloria Confessorum de Grégoire, placé à la fin de chacune des deux Vitae, convient parfaitement : il apparaît comme la suite logique de celles-ci. En parlant de la redécouverte du corps d'Ursin et en évoquant brièvement ses miracles post mortem, il prend le relais avec la scène de la mort d'Ursin qui achève le texte des Vitae et semble, de cette manière, clore la narration ${ }^{29}$. Ainsi, la vie d'Ursin se déroule très nettement

22. Catalogue général des manuscrits des bibliothèques publiques de France. Départements, tome IV, Paris, 1886, p. 81.

23. Je remercie ici $M^{\text {me }}$ Marie-Jeanne BOISTARD, conservatrice de la Bibliothèque des Quatre-Piliers, bibliothèque municipale de Bourges, pour m'avoir fourni cette information.

24. Grosjean, Paul, "Catalogus codicum Hagiographicorum latinorum bibliothecarum edinburgensium ", dans Analecta Bollandiana, XLVII, 1929, I et II, p. 31-38.

25. AigRAin, René, L'Hagiographie, ses sources, ses méthodes, son histoire, Paris, 1953, p. 186 sqq. : René Aigrain faisait remarquer que « les récits de translations ou d'inventions des reliques figur(aient) parfois en conclusion des Vies de saints, mais constitu[aient] fréquemment des compositions indépendantes".

26. BEAUJARD Brigitte, Le culte des martyrs en Gaule d'Hilaire de Poitiers à la fin du VI siècle, tome 1, Thèse de doctorat d'État sous la direction de Luce PIETRI, Paris, Université de Paris IV, 1993, p. 414, note 427 : Le Livre à la Gloire des Confesseurs aurait définitivement été achevé entre 587 et 591.

27. Delehaye H., " De sancto Ursino, primo biturigensi episcopo », dans op. cit., p. 102

28. VAN DER StRAeten, Joseph, op. cit., p. 62-63.

29. On pourra consulter au sujet de cette idée l'article de LAUwERS, Michel, « La mort et 
en trois temps : la période de sa naissance et de sa vie auprès du Christ et des apôtres, puis sa mission d'évangélisation à Bourges jusqu'à sa mort et enfin, l'épisode de l'invention et de la translation de son tombeau ${ }^{30}$.

En ce qui concerne les trois épitomés, le BHL 8414 apparaît dans l'appendice de la Légende dorée (Legenda aurea) publiée en 1507 (chapitre 192) et en 1518 (chapitre 193) ${ }^{31}$. Il s'agit d'un abrégé de la Vita BHL 8413 tout comme l'épitomé BHL 8415 publié en 1757 par Philippe Labbe dans sa Nova Bibliotheca $^{32}$. En outre, l'épitomé BHL 8415 b est connu par l'intermédiaire d'un manuscrit qui était conservé dans l'église du Saint-Sauveur à Utrecht, maintenant devenue la Bibliothèque universitaire de la ville : le texte se présente sous la forme d'une vie très courte d'Ursin, ne s'étendant que sur quelques lignes ${ }^{33}$. L'hagiographe, s'éloignant, dans ce texte, des autres Vitae, s'emploie uniquement à rapporter qu'Ursin et le disciple Nathanaël, frère de l'apôtre Philippe, n'étaient qu'une seule et même personne.

Une étude attentive de la Vita A et de la Vita B (BHL 8412 et BHL 8413) montre que le texte de celles-ci est extrêmement proche à quelques détails près. En effet, elles nous apportent des informations identiques sur la vie du premier évêque de Bourges. Si, dans le texte de la Vita B, l'hagiographe commence son récit à la naissance d'Ursin, ajoutant dans le prologue qu'avant son baptême, il était appelé Nathanaël ${ }^{34}$, et poursuit en narrant la vie du saint auprès du Christ et des apôtres jusqu'à son départ pour Rome en compagnie de Pierre (leçons 1, 2 et la moitié de la 3), les deux textes se rejoignent au moment où le premier évêque de Bourges est envoyé en mission dans les Gaules. Un seul élément différencie les deux récits sur ce point : la Vita B précise qu'Ursin aurait été envoyé par le pape Clément lui-même, tandis que le texte de la Vita A indique seulement que le saint aurait reçu sa mission des saints apôtres ${ }^{35}$ sans autre précision. Les événements qui suivent son arrivée dans le Berry, comme son exil hors de la ville, sont très abrégés dans la Vita A en comparaison avec le texte de la Vita B. En outre, le passage le plus développé par l'auteur de la Vita A est sans conteste celui

le corps des saints. La scène de la mort dans les Vitae du haut Moyen Âge ", dans Le Moyen Âge, XCIV (5 ${ }^{\mathrm{e}}$ série. t. 2), 1988, n 1 , p. 21-50, particulièrement p. 22.

30. Le Père Hippolyte DELEHAYE rappelle dans ses Légendes hagiographiques (Bruxelles, 1927, p. 92) que c'est la construction classique d'une Vie de saint.

31. Bibliotheca Hagiographica Latina ..., op. cit., p. 1216.

32. LABBE, Philippe, op. cit., p. 459-460.

33. Deux manuscrits possédant l'épitomé ont été recensés par les Bollandistes (Delehaye, Hippolyte, " De sancto Ursino... ", dans Delehaye, Hippolyte, PeETERS, Paul [éd.], $o p$. cit., p. 103). Le premier est conservé à la Bibliothèque royale de Belgique (cod. Bruxellensis, 8936-8938, $\mathrm{f}^{\circ}$ 112-116) et le second, copié sur le codex bruxellois, à la bibliothèque universitaire d'Utrecht.

34. "Hic enim Nathanahel esse dicitur de quo ipsa Veritas sanctitatis testimonium perhibens dixit : "Ecce vere Israhelita in quo dolus non est". Verum postea baptizatus ab apostolis Ursinus est appellatus sicut de Paulo apostolo legitur, qui ante conversionem Saulus dicebatur, et postquam adeptus vere fidei professionem fuit, Paulus est vocatus. " (BHL 8413) : Acta Santorum, Novembris, Tomus IV, p. 109.

35. "...qui a sanctis apostolis ab urbe Roma [...], Evangelii semina sparsurus Galliis directus fuisset... " (BHL 8412) : FAILLON, Étienne-Michel, op. cit., col. 423. 
du don fait par Leocadius, sénateur des Gaules, dans un premier temps de ses écuries, et, dans un second, de son palais. Toutefois, on observe qu'aucune phrase de la Vita A, à la différence de la Vita B, ne dit qu'Ursin fut le compagnon d'Étienne dont il aurait recueilli le sang à sa lapidation : l'hagiographe cite seulement les reliques du martyr lors de la dédicace des deux églises consacrées au saint. Par ailleurs, dans cette dernière Vie, n'apparaît pas non plus un long fragment présent dans la Vita B : il s'agit du sermon prononcé par Ursin à Leocadius pour le convaincre de se convertir au christianisme (leçon 8), sermon juste mentionné dans le premier récit. Un autre élément est absent de la Vita A : le prologue de quelques lignes qui constitue le premier paragraphe de la Vita B. L'hagiographe, exposant sa méthode et se défendant de toute compétence, loue la figure de saint Ursin. Pour terminer, les deux textes s'achèvent d'une manière analogue : Leocadius offre toutes ses propriétés et tous ses biens dans le Berry à l'Église de Bourges et à son premier représentant, Ursin. Identique est aussi l'épisode de la mort du saint précédé de l'institution de son successeur, Sénécien.

Il est facile d'observer que les textes des deux Vitae sont en de nombreux points tout à fait semblables, ce qui laisse supposer que l'un a servi de modèle à l'autre. Par exemple, les précisions données au sujet de la mort du compagnon du saint, Just, sont identiques dans les deux textes ${ }^{36}$. Certaines phrases citées dans chacune des Vies sont extraites des mêmes livres bibliques : comme cette sentence issue de l'Évangile de Jean, "S'ils m'ont persécuté, ils vous persécuteront aussi " (Jn 15, 20), placée dans la Vita $\mathrm{B}$ à la fin de la leçon 4 et présente de manière littérale dans la Vita $\mathrm{A}^{37}$. On finit par constater que la Vita B (BHL 8413) n'est autre qu'une version plus étoffée du texte de la Vita A (BHL 8412) : toutes les scènes sont volontairement plus détaillées à commencer par le récit de la naissance d'Ursin. Il est très vraisemblable que la Vita A ait pu servir de modèle pour l'hagiographe à l'origine de la Vita B, qui n'aurait fait qu'appliquer la « méthode d'amplification ", cherchant délibérément à développer le récit ${ }^{38}$. Le sermon prononcé par Ursin à Leocadius en serait d'ailleurs un parfait exemple. Ou peut-on imaginer que l'inverse se soit produit? La Vita A pourrait-elle

36. "Hic namque Justus cum beato Ursino, ad urbem Biturigam properans, orientali in urbis plaga, miliario ab urbe nono, super alveum Utrionem feliciter mligravit ad Christum. " (BHL 8412) : FAILLN, Étienne-Michel, op. cit., col. 423; "Qui etiam miliario ab urbe non in orientali plaga super Utrionis alveum ultimum claudens diem feliciter migravit ad Christum. " (BHL 8413) : Acta Santorum, Novembris, Tomus IV, p. 110.

37. "Si me persecuti sunt et vos persequentur" (BHL 8412 et 8413) : respectivement, FAILlon, Étienne-Michel, op. cit., col. 423 et : Acta Santorum, Novembris, Tomus IV, p. 111.

38. Delehaye, Hyppolite, Les Légendes..., op. cit., p. 92. Voir également un ensemble de publications récentes ayant pour thème central d'étude la question de la réécriture des vies de saints : Goullet, Monique et HeInZElmann, Martin, (dir.), La réécriture hagiographique dans l'Occident médiéval, Transformations formelles et idéologiques, Ostfildern, 2003; Le riscritture agiografiche, $v$ Convegno Internazionale della SISMEL, Firenze, Certosa del Galluzzo, 22-23 mars 2002, (publié dans Hagiographica, X, 2003, p. 107-297); GouLLET, Monique, Écriture et réécriture hagiographiques. Essai sur les réécritures de Vies de saints dans l’Occident latin médiéval (VIII'-XII' siècle), (Hagiologia, 4), Turnhout, 2005. 
être un résumé de la Vita $\mathrm{B}$ ? La tradition a eu néanmoins tendance à considérer la Vita A comme la Vie la plus ancienne ${ }^{39}$.

En effet, le plus vieux manuscrit conservé de la Vita A est daté des $\mathrm{X}^{\mathrm{e}}$ $\mathrm{XI}^{\mathrm{e}}$ siècles, même si les folios nous intéressant semblent appartenir au XI $\mathrm{e}^{\mathrm{e}}$ siècle ${ }^{40}$. Les autres manuscrits possédant cette Vie s'échelonnent ensuite entre le XI ${ }^{\mathrm{e}}$ et le $\mathrm{XII}^{\mathrm{e}}$ siècle, avec une copie au XV siècle. Quant à la Vita $\mathrm{B}$, on constate que la majorité des manuscrits transmettant ce texte sont du $\mathrm{XI}^{\mathrm{e}}$ siècle, à l'exception de l'un d'entre eux daté du XIII ${ }^{\mathrm{e}}$ siècle ainsi qu'une copie datant du Xve siècle. Cette brève synthèse chronologique permet de constater que le premier témoignage aujourd'hui possédé de la Vita A est conservé dans un manuscrit plus ancien que celui de la Vita B. Mais tirer des conclusions à partir de cette seule observation serait trop simple. Elle met aussi en évidence le fait que l'ensemble des manuscrits conservant des Vitae d'Ursin sont postérieurs au haut Moyen Âge. Seul le récit de la revelatio, inséré dans le chapitre LXXIX du Liber in Gloria Confessorum de Grégoire de Tours, se rattache à cette période et est contemporain de la redécouverte du corps ${ }^{41}$.

Dans ces circonstances, il est légitime de s'interroger sur l'origine textuelle des deux Vitae : ont-elles été rédigées pour la première fois au Moyen Âge central ou s'appuient-elles sur une version primitive qui aurait pu être composée avant le $\mathrm{x}^{\mathrm{e}}$ siècle et dont on aurait perdu le manuscrit original? Passons à présent à l'observation rapprochée des deux textes qui doit nous aider dans cette tentative de datation.

\section{Forme hagiographique des Vitae}

Les paragraphes précédents nous ont permis de formuler quelques remarques sur le corpus hagiographique de saint Ursin. Les différences séparant les deux Vitae ont été énoncées pour mieux mettre en évidence la grande similitude qui existe entre les deux textes, tant du point de vue du contenu que de celui de la forme ${ }^{42}$. En s'appuyant sur cette proximité textuelle, les observations faites dans ce chapitre sont donc valides pour chacune des Vies. Témoignage de l'hagiographie médiévale, chaque Vita est construite selon des procédés stylistiques récurrents utilisés par les auteurs de l'époque, procédés qu'on tente ici de déceler. Le but est égale-

39. DeleHAYE, Hyppolite, « De sancto Ursino, primo biturigensi episcopo », dans op. cit., p. 102. 40. Il s'agit d'un recueil composite (BNF, ms. lat. 13220) dont la datation est extrêmement difficile. Après consultation des folios 13 à 19 conservant la Vita A de saint Ursin, il semblerait que ceux-ci puissent être datés du XI ${ }^{\mathrm{e}}$ siècle. Seuls les folios 52 à 59 contenant les Sermones in natale $s$. Martialis, reconnus comme étant de la main d'Adémar de Chabannes (989-1034), sont datés entre 1029 et 1034 : SAMARAN, Charles et MARICHAL, Robert, op. cit., p. 319. Un grand remerciement à $\mathrm{M}^{\mathrm{me}}$ Marie-Françoise DAMONGEOT, conservateur en chef au département des manuscrits de la BNF, pour m'avoir permis de consulter le manuscrit.

41. La redécouverte du corps de saint Ursin daterait, si on s'appuie sur le chapitre LXXIX du Liber in Gloria Confessorum de Grégoire de Tours, du milieu du VI ${ }^{\mathrm{e}}$ siècle : cf. LE LUEL, Nathalie, La légende de fondation de l'église Saint-Ursin de Bourges..., op. cit., p. 79-90.

42. On constate d'ailleurs qu'elles, toutes deux, appartiennent au type biographique des vies de saint (cf. Goullet, Monique, op. cit., p. 212). 
ment de pointer du doigt les clichés hagiographiques les plus caractéristiques ressortant des écrits sur le saint.

À la lecture des deux textes construits tous deux selon un plan chronologique ${ }^{43}$, on constate le très grand nombre de citations ou de paraphrases de la Bible utilisées par les hagiographes, plus particulièrement encore dans la Vita B du fait de sa longueur. On les repère d'ailleurs aisément dans le texte publié par les Bollandistes. La majorité de ces phrases est tirée du Nouveau Testament : des Évangiles (la plupart de ceux de Matthieu et de Jean, moins de Luc et de Marc), des Actes des apôtres, des épîtres (épître aux Romains, première épître aux Corinthiens, première épître à Timothée, épître à Tite). Quelques citations sont aussi extraites de l'Ancien Testament, surtout du Livre des Psaumes, mais également du Livre de la Genèse, dans le sermon fait à Leocadius, et enfin du Libre de Job (uniquement dans la Vita B). Une telle organisation du texte, qui s'appuie sur des références bibliques pour donner de l'autorité au récit, est un procédé hagiographique typique qu'on retrouve dans les trois épitomés mais aussi dans la plupart des Vitae de saints ${ }^{44}$. Ce recours « direct et formel " à la Bible, et ceci de manière abondante, est caractéristique des biographies hagiographiques " écrites très longtemps après la mort du saint " et pour lesquelles existe un " manque d'informations concrètes " conduisant inévitablement à l'accroissement du nombre des topoï littéraires ${ }^{45}$. Par ailleurs, le discours prononcé par Ursin devant Leocadius est un parfait exemple de ce type de "sermons presque entièrement faits de morceaux " bibliques destinés à " inculquer [aux] lecteurs et auditeurs l'amour de l'Écriture sainte et de son étude dévouée ${ }^{46}$ ".

C'est ainsi que de nombreux clichés hagiographiques apparaissent dans les deux Vitae. Ils ne sont pas uniquement propres à l'hagiographie de saint

43. Sur cette question, SigAL, Pierre-André, " Le travail des hagiographes aux XI et XII siècles : sources d'information et méthodes de rédaction ", dans Francia, vol. 15, 1987, p. 149-182 et plus spécialement p. 177 sqq.

44. Jean LECLERCQ, dans son article intitulé « L'écriture sainte dans l'hagiographie monastique du haut Moyen Âge ", paru en 1963 (La Bibbia nell'alto medioevo, Settimane di Studio del Centro italiano di Studi sull'alto Medioevo, 26 aprile-2 maggio 1962, Spolète, 1963, p. 103128), constate que, dans la littérature légendaire, les livres « qui sont cités le plus abondamment sont le Psautier et les quatre Évangiles " (p. 107). On pourra se référer sur ce point également aux travaux de Marc VAN UYTFANGHE, "Le culte des saints et l'hagiographie face à l'écriture : les avatars d'une relation ambiguë », dans Santi e Demoni nell'alto medioevo occidentale (secoli V-XI), Settimane di Studio del Centro italiano di Studi sull'alto Medioevo, 7-13 aprile 1988, Spolète, 1989, p. 155-202.

45. VAn UytFanghe, Marc, "Modèles bibliques dans l'hagiographie ", dans Riché, Pierre et Lobrichon, Guy (dir.), Le Moyen Âge et la Bible, Paris, 1984, p. 449-488 et plus particulièrement p. 454; id., "Le remploi dans l'hagiographie : une "loi du genre " qui étouffe l'originalité? ", dans Ideologie e pratiche del reimpiego nell'alto medioevo, Settimane di studio del centro italiano di studi sull'alto medioevo, XLVI, 16-21 aprile 1998, vol. 1, Spolète, 1999, p. 359-411 et surtout p. 391. Voir aussi à ce sujet, même si l'objet de l'étude précède notre propos : id., Stylisation biblique et condition humaine dans l'hagiographie mérovingienne, 600-750, Bruxelles, 1987, notamment p. 60.

46. VAn UytFanghe, Marc, "Modèles bibliques dans l'hagiographie ", dans Riché, Pierre et LOBRICHON, Guy (dir.), op. cit., p. 464. 
Ursin mais à l'ensemble du genre littéraire. Un autre exemple en est le début du texte de la Vita B, cliché des origines apostoliques ${ }^{47}$, l'hagiographe ayant choisi de faire naître Ursin à l'époque du Christ et de lui faire côtoyer les apôtres ${ }^{48}$. Le prologue rédigé par celui-ci est également tout à fait caractéristique de cette littérature légendaire : l'auteur y conserve l'anonymat et utilise le motif classique de la modestie affectée, de l'humilité pour introduire son texte ${ }^{49}$. Il y affiche également très clairement ses intentions et la méthode employée pour parvenir à une composition honorable, encourageant le peuple à suivre le modèle du saint évêque.

Attardons-nous également sur le cas de la mission des Sept évêques ${ }^{50}$ envoyés de Rome en Gaule ${ }^{51}$ et évoquée dans les deux Vitae. Dans la B, Ursin est placé en tête des noms énoncés pour cette mission, qui n'est donc plus composée de sept évêques mais de huit. Dans la Vita A, la position d'Ursin au sein de la mission est moins affirmée. Elle se rapproche davantage de la place donnée au saint dans le chapitre XXXI de l'Historia Francorum de Grégoire de Tours.

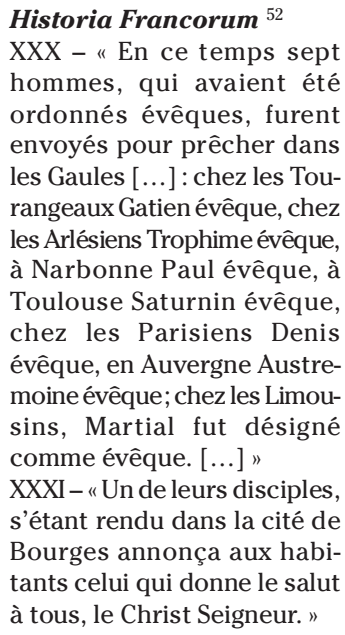

\section{Vita A ${ }^{53}$}

"Le très saint Ursin [...] avait été envoyé, par les saints apôtres depuis la ville de Rome, répandre la semence de l'Évangile dans les Gaules, avec le très précieux sang du protomartyr du Christ Etienne, et les compagnons qui furent saint Denis de Paris, saint Saturnin de Toulouse, saint Trophime d'Arles, saint Paul de Narbonne..., saint Austremoine d'Auvergne et saint Gatien évêque..."

\section{Vita $\mathbf{B}^{54}$}

"Le bienheureux Clément, ayant reçu la charge de l'Église et soucieux d'appliquer ce que Pierre lui avait recommandé pour que la foi catholique soit divulguée et propagée un peu partout par une fidèle prédication, choisit des prédicateurs habiles à parler et constants dans la foi de toute l'Église, les très saints Ursin, Denys l'Aréopagite [...], Martial, Saturnin, Trophime, Paul, Austremoine et Gatien. "

47. Sur ce point, voir Houtin, Albert, La controverse de l'apostolicité des églises de France,

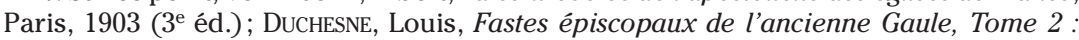
L'Aquitaine et les Lyonnaises, Paris, 1910 (2e éd.). Plus récemment, voir à ce sujet La mémoire des origines dans les institutions médiévales, Actes de la table ronde organisée à l'École française de Rome en juin 2002, dans Mélanges de l'EFR, tome 115, 2003, 1, p. 132- 267.

48. DelehaYe, Hyppolite, Les légendes..., op. cit., p. 52-53.

49. Ibidem, p. 63. Voir également l'article plus récent de Pierre-André SigAL, «Le travail des hagiographes aux XI ${ }^{\mathrm{e}}$ et XII ${ }^{\mathrm{e}}$ siècles... ", dans op. cit., p. 151, 156 et 171.

50. Gatien (Tours), Trophime (Arles), Paul (Narbonne), Saturnin (Toulouse) et Denis (Paris).

51. À ce sujet, se référer à LEVILLAIN, Léon, "Saint Trophime et la mission des sept en Gaule ", dans $R H E F$, XIII, 1927, p. 145-189 et, pour un bilan plus récent sur la question, consulter BEAUJARD, Brigitte, op. cit., p. 389 sqq.

52. «30. Huius tempore septem viri episcopi ordenati ad praedicandum in Galliis missi sunt [...] : Turonicis Catianus episcopus, Arelatensibus Trophimus episcopus, Arvernis 
Cette image d'une mission d'évangélisation commandée par les apôtres eux-mêmes n'est pas propre à l'hagiographie de la Gallia. On la retrouve par exemple dans l'hagiographie de la Péninsule ibérique où il est également raconté que sept disciples de saint Jacques, choisis à Rome par saint Pierre, auraient été envoyés en Péninsule ibérique porter l'Évangile ${ }^{55}$. Grégoire et ensuite les hagiographes du Moyen Âge central ont simplement été les vecteurs d'une légende, d'origine probablement orale, qui s'est diffusée dès le haut Moyen Âge.

En avançant dans l'histoire d'Ursin, un autre motif hagiographique apparaît lorsque le compagnon du saint, du nom de Just, meurt en route après avoir franchi les frontières du Berry à peu de distance de la ville de Bourges. Ursin est alors en proie au désespoir et au doute ne sachant s'il doit continuer sa mission. Suivant un schéma classique, on retrouve le même type d'épisode dans nombre de légendes de saints ${ }^{56}$ : c'est le cas dans les Vies de saint Martial (BHL 5551-5552) mais, à la différence d'Ursin, l'évêque de Limoges ressuscite son disciple saint Austriclinien ${ }^{57}$ tout comme saint Front (BHL 3182) avec son compagnon Georges. Ce dernier, au bout de trois jours de voyage qui le menait en Gaule au côté de l'évêque de Périgueux, meurt mais Front, après être retourné vers Pierre plein de tristesse, le ressuscite " par la vertu du bâton pastoral " qui lui avait été confié par le premier des apôtres ${ }^{58}$. La scène est pratiquement identique à celle des Vies de saint Martial.

Stremonius episcopus, Lemovicinis Martialis est distinatus episcopus. [...] 31. De horum vero discipulis quidam Bituricas cvitatem adgressus, salutare omnium, Christum dominum populis nuntiavit. " : "Gregorii episcopi Turonensis Historiarum Liber I ", dans op. cit., p. 22-24. Traduction de Robert LatouchE : cf. Grégoire de Tours, Histoire des Francs, tome 1, Paris, 1963, Livre I, p. 54-57.

53. « Sanctissimus [...] Ursinus, qui a sanctis apostolis ab urbe Roma, cum pretiosissimo protomartyris Christi Stephani sanguine, comitibusque qui sunt sanctus Dionysius Parisiaciensis, sanctus Saturninus Tholonensis, Trophimus Arelatensis, Paulus Narbonensis, [...], Austremonius Arvernensis, et sanctus Vatianus episcopus, Evangelii semina sparsurus Galiis directus fuisset..." (BHL 8412) : FAlLLON E. M., op. cit., col. 423.

54. " Suscepta igitur beatus Clemens ecclesie cura, sollicitus eorum que sibi a Petro fuerant commendata, ut fides catholica per orbis esset elimata predicatione fidelium propalata, predicatores disertos et constantes in fide ex tota assumpsit ecclesia beatissimum Ursinum, Dionisium Atheniensem [...], Martialem, Saturninum, Trophimum, Paulum, Austremonium, ac Gatianum et eorum discipulos... " (BHL 8413) : Acta Santorum, Novembris, Tomus IV, p. 110.

55. KuRTH, Geoffroy, «Les traditions du vi ${ }^{\mathrm{e}}$ siècle sur l'apostolicité de saint Denis de Paris ", dans Kurth G., Etudes Franques, t. 2, Paris/Bruxelles, 1919, p. 302 : l'historien les nomme Torquatus à Guadix, Eufrasius à Andujar, Ctesifon à Berja, Indalecius à Almeria, Secundus à Abula, Caecilius à Grenade et Hesychius à Cazorla; voir aussi LevILLAIN, Léon, "Saint Trophime et la mission des sept en Gaule ", dans op. cit., p. 167 et LAUGARDIÈRE, Maurice de, L'Église de Bourges avant Charlemagne, Paris, 1951, p. 16, note 3.

56. VeiLlat, Just, « Légende de saint Ursin, patron du Berry, de saint Léocade et de saint Ludre ", dans Comptes-rendus des travaux de la société du Berry à Paris, vol. Ix, 1862, p. 128168, plus spécialement p. 139, note 1. Voir également DuCHESNE, Louis, op. cit., p. 108-109. 57. Saint Martial avait deux compagnons, saint Alpinien et saint Austriclinien.

58. CoENS, Maurice, "La vie ancienne de saint Front ", dans Analecta Bollandiana., XLVIII, 1930, p. 324-360, spécialement p. 324 et 328 : Georges serait ensuite resté dans le Velay, épuisé, et y aurait fondé l'Église du Puy. 
La manière dont se déroule l'évangélisation de Bourges par Ursin appartient également aux modèles hagiographiques utilisés par les clercs de l'époque. Le saint entre dans la ville et commence à prêcher, viennent à lui tout d'abord les plus pauvres puis les puissants. Mais les Gentils, prenant peur, chassent le saint qui, après une phase de doute, revient encore davantage confiant dans la nécessité de sa mission et convertit de grandes foules. Cette organisation correspond au schéma d'évangélisation de nombreux saints, en particulier l'épisode de l'exil qui est très souvent raconté et qui, à la base et dans cette volonté d'imitation, est calqué sur la vie du Christ ${ }^{59}$. La puissance divine est encore une fois soulignée lors de la conversion de Leocadius, sénateur des Gaules, par Ursin. L'hagiographe insiste particulièrement sur le fait que le sénateur était païen mais pieux en actes. Leocadius joue un rôle bien précis au sein de la légende d'Ursin : il est censé illustrer, d'un côté, la conversion d'un haut fonctionnaire du pouvoir romain et, de l'autre, la supériorité de la religion chrétienne sur la religion païenne grâce à l'action de son serviteur Ursin. De cette manière, on fait de Leocadius un exemple de conversion au christianisme et d'obéissance. Du reste, le fait que Leocadius soit mêlé à la christianisation de Bourges est caractéristique de l'influence qu'eut le milieu aristocratique gaulois d'origine romaine sur les débuts du christianisme en Gaule et dont Grégoire de Tours est le digne héritier ${ }^{60}$.

Les miracles accomplis par Ursin, aussi brièvement évoqués soient-ils dans les Vitae, sont des topoï de l'hagiographie : ils sont copiés sur ceux qu'accomplit le Christ dans le Nouveau Testament, ultime référence en matière légendaire. En effet, Ursin rend la vue aux aveugles mais il guérit aussi les paralytiques et les boiteux ${ }^{61}$.

L'épisode de la mort de saint Ursin est, encore une fois, construit selon un plan très précis propre à l'hagiographie. C'est Dieu lui-même pour récompenser le saint de sa peine qui lui fait ressentir par une douleur corporelle, souvent une fièvre comme ici d'ailleurs, que sa fin est proche ${ }^{62}$. Il s'agit donc d'une mort pressentie plus qu'annoncée. Sentant ses forces l'abandonner, le saint prépare alors son départ en réunissant la commu-

59. VAn UytFanghe, Marc, (" Modèles bibliques dans l'hagiographie », dans RichÉ, Pierre et LOBRICHON, Guy, (dir.), op. cit., p. 464) observe que le saint ne réplique pas à ces " humiliations et $[\ldots]$ souffrances forcées".

60. BEAUJARD, Brigitte, op. cit., tome 1, p. 169. Voir également à ce propos, même si cela concerne davantage la christianisation dans le Nord-Est de la Gaule : WERNER, KarlFerdinand, "Le rôle de l'aristocratie dans la christianisation du Nord-Est de la Gaule ", dans La christianisation des pays entre Loire et Rhin (IV ${ }^{e}$-VIf siècles), Actes du colloque de Nanterre, $R H E F$, LXII, $\mathrm{n}^{\circ} 168$, janvier-juin 1976, p. 45-73 ainsi que HEINZELMANN, Martin, "L'aristocratie et les évêchés entre Loire et Rhin jusqu'à la fin du VII siècle ", dans $L a$ christianisation des pays entre Loire et Rhin..., op. cit., p. 75-90.

61. Delehaye, Hyppolite, Les Légendes..., op. cit., p. 92.

62. LAUWERS, Michel, "La mort et le corps des saints... ", dans op. cit., p. 23, 27 et 42 ; concernant les différents schèmes composant classiquement une vie de saint, notamment celle de la " prescience de la mort ", on se référera à Goullet, Monique, op. cit., p. 213. 
nauté des fidèles ${ }^{63}$ et en instituant un successeur, étapes que suit à la lettre Ursin. Donner la date du trépas du saint est aussi un lieu commun hagiographique $^{64}$ : dans le cas d'Ursin, il s'agit du 4 des calendes de janvier. Ce passage dans l'au-delà n'est pas montré comme un moment douloureux mais comme un instant où la sérénité et la joie sont de mise ${ }^{65}$, " il migra par un décès heureux dans la patrie d'Abraham " dans la Vita $\mathrm{A}^{66}$ ou encore " il fut transporté, un chœur d'anges chantant, dans la joie du Paradis" dans la Vita $\mathrm{B}^{67}$, état qui contraste avec les pleurs des fidèles. On remarque du reste que la mort n'est évoquée que par l'intermédiaire de périphrases qui ont pour fonction de dédramatiser cet événement qui effrayait tant l'homme médiéval ${ }^{68}$.

Enfin, Ursin n'est pas le seul à être devenu un des soixante-douze disciples du Christ au point que l'auteur de la Vita B et celui du texte BHL 8415b l'a très intentionnellement confondu avec le Nathanaël de l'Évangile $^{69}$ : c'est aussi le cas de saint Front de Périgueux (BHL 3182) ${ }^{70}$ ou encore de saint Denis de Paris (BHL 2171) identifié avec Denys l'Aéropagite. Tous les trois, d'après leur légende, furent envoyés en Gaule par saint Pierre ou saint Clément pour évangéliser le territoire, tradition toujours dans la droite veine de la thèse apostoliciste.

À ce stade de l'étude, interrogeons-nous sur les sources à l'origine des Vitae A et B d'Ursin. Où les hagiographes ont-ils été puisé? On constate très facilement que rien de ce que narre Grégoire de Tours dans le chapitre XXXI de son Historia Francorum n'est absent des deux Vitae d'Ursin à l'exception du fait que Leocadius était un descendant du martyr lyonnais Vettius Epagathus. L'opinion, très largement admise aujourd'hui, est que ce chapitre serait une des sources utilisées par les hagiographes d'Ursin ${ }^{71}$. Le

63. LAUWERS, Michel, « La mort et le corps des saints... ", dans op. cit., p. 24-25.

64. Ibidem, p. 30 et 36.

65. Ibid., p. 26.

66. "...Abrahae patriam felici migravit excessu. " (BHL 8412)

67. "...angelorum psallentibus choris translatus est ad gaudia paradis... " (BHL 8413)

68. LAUWERS, Michel, "La mort et le corps des saints... ", dans op. cit., p. 29 et 32-33 : Michel LAUWERS écrit que " cette vision pacifiée de la mort témoigne de la transformation de la sainteté : à l'époque mérovingienne, le modèle des saints évêques et des saints abbés se substitue à celui des martyrs et des confesseurs. Plutôt que le martyre qui ne correspond plus guère aux possibilités de sanctification du moment, la représentation du saint mort dans la paix devient un exemple. "

69. Nathanaël est un disciple du Christ mentionné dans l'Évangile de Jean, (Jean, I, 4351 et XXI, 2). L'évangéliste nous apprend que Nathanaël aurait été présenté par Philippe à Jésus après qu'il l'a vu sous le figuier. Il aurait également assisté à l'apparition du Christ sur les bords du lac de Tibériade en compagnie de Simon-Pierre, Thomas, des fils de Zébédée ainsi que de deux autres disciples.

70. CoEns, Maurice, "La " scriptura de sancto Fronto Nova " attribuée au chorévêque Gauzbert ", dans Analecta Bollandiana, LXXV, 1957, 3 et 4, p. 340-356 et surtout p. 341.

71. ChEVAlier, Abbé C., "Les origines de l'Église de Tours ", dans Mémoires de la Société archéologique de Touraine, tome XXI, 1871, p. 69-634, mais particulièrement p. 139-143 et 474-480; KURTH, Geoffroy, "De l'autorité de Grégoire de Tours ", dans op. cit., p. 139; DeleHAYE, Hyppolite, « De sancto Ursino, primo biturigensi episcopo », dans op. cit., p. 102. 
texte de la Vita A ne serait qu' " une amplification maladroite [...] d'un récit plus simple et véridique ${ }^{72} "$; on peut supposer qu'il s'agit de celui de Grégoire de Tours. Notons que ces remarques s'appliquent tout autant à la Vita B. Dans l'épitomé BHL 8415b, c'est encore, et ici sans la moindre objection possible, à l'évêque tourangeau que l'hagiographe, ayant confondu Nathanaël et Ursin, a emprunté la fin de son passage. Cependant, cette fois, ce n'est pas à l'Historia Francorum que le texte fait référence mais au chapitre LXXIX du Liber in Gloria Confessorum.

Se dressant contre cette opinion des origines apostoliques de l'Église de France réapparue au XIX ${ }^{\mathrm{e}}$ siècle, l'abbé Casimir Chevalier fut le premier à déplacer vers le Moyen Âge central la datation des vies d'Ursin après avoir remarqué que l'hagiographe de la Vita B avait imité un passage de la légende de saint Martial composée à la fin du $\mathrm{x}^{\mathrm{e}}$ siècle ou au début du $\mathrm{XI}^{\mathrm{e}}$ siècle ${ }^{73}$. Toute la partie qui fait d'Ursin un contemporain du Christ et des apôtres, le faisant assister à la Cène, au lavement des pieds, à la Passion du Seigneur, à sa Résurrection, à l'Ascension et à la Pentecôte jusqu'à suivre saint Pierre à Rome, utilise un schéma identique à la Vita de saint Martial, attribuée au Pseudo-Aurélien (BHL 5552) ${ }^{74}$. La similitude entre les deux Vitae de l'épisode de la résurrection du compagnon de voyage ajoute encore à cette ressemblance entre les deux textes ${ }^{75}$. Quant au chanoine de Laugardière, il faisait à son tour remarquer les liens avec la Vita tertia de saint Austremoine (848) ${ }^{76}$ : en effet, est attribué un même début de carrière au saint d'Auvergne qu'à Martial et Ursin, témoignages encore une fois de la rhétorique hagiographique propre au cliché des origines apostoliques. Par ailleurs, selon H. Delehaye commentant la Vita B d'Ursin, en plus de

72. Chevalier, Abbé C., " Les origines de l'Église de Tours ", dans op. cit., p. 139.

73. Ibidem, p. 144, note 1, et 476-478. Il existe plusieurs légendes de Martial dont la première date du IX ${ }^{\mathrm{e}}$ siècle mais ici c'est précisément à la Vita de la fin $\mathrm{X}^{\mathrm{e}}$ siècle/début $\mathrm{XI}^{\mathrm{e}}$ siècle (BHL 5552), attribuée à Aurélien, compagnon du saint, que l'hagiographe de la Vita B d’Ursin a emprunté des éléments. Pour Pierre Bonnassié (Bonnassié, Pierre, Sigal PierreAndré, Iogna-Prat Dominique, "La Gallia du Sud, 930-1130 ", dans PhILIPPART, Guy (dir.), Hagiographies, Histoire internationale de la littérature hagiographiques latine et vernaculaire en Occident des origines à 1550, vol. 1, Turnhout, 1994, p. 289-344, et plus particulièrement p. 297), la Vita aurait été écrite entre 994 et 1028, date de la consécration de la nouvelle basilique Saint-Martial. Seule différence avec Ursin, Martial aurait été le propre neveu de saint Pierre.

74. On pourra consulter DuCHESNE, Louis, op. cit., p. 111. Sur la question de la filiation entre certaines vies de saint, on pourra se référer à l'article de Baudouin de GAIFFIER, "Les thèmes hagiographiques; est-il possible d'établir pour chacun d'eux une filiation? ", dans Revue d'Histoire ecclésiastique, LXXVII, 1982, p. 78-81.

75. Poulin, Joseph-Claude, L'idéal de sainteté dans l'Aquitaine carolingienne d'après les sources hagiographiques (750-950), Québec, 1975, p. 61 : l'auteur ajoute que le thème du " compagnon de route " ressuscité par le bâton de saint Pierre est "un thème peut-être introduit en Gaule par le biographe de saint Front ", thème qui fut " abondamment diffusé à partir de la seconde moitié du xe siècle par la légende aurélienne ".

76. LAUGARDIÈRE, Maurice de, op. cit., p. 18 et 21 : précisons également que le chanoine reconnaissait dans la légende d'Ursin " un arrangement des récits de Grégoire ". Il faisait référence ici à la troisième vie de saint Austremoine rédigée comme les autres dans l'abbaye de Mozat : celle-ci daterait du début $\mathrm{XI}^{\mathrm{e}}$ siècle. 
s'être servi des livres bibliques et d'avoir utilisé très librement l'Historia Francorum de Grégoire, l'hagiographe aurait aussi puisé dans les annales liturgiques de la ville de Bourges ${ }^{77}$. Un même chemin fut sans doute suivi par l'auteur de la Vita A. La présence d'un disciple du nom de Just est ajoutée, l'hagiographe de la Vita A ou celui de la Vita B s'appuyant sur la dévotion qu'un village voisin de Bourges avait pour le saint. Aurait également été inventé l'épisode d'un premier sanctuaire établi dans les écuries du sénateur Leocadius. On aurait introduit, dans le récit, la présence d'un fils aîné de Leocadius, Caremuselus, et le don perpétuel fait par le sénateur des Gaules, après son baptême, à l'Église de Bourges de l'ensemble de ses domaines berrichons ${ }^{78}$. En plus de cela, l'auteur de la Vita $\mathrm{B}$ aurait imaginé qu'Ursin avait lui-même apporté les reliques de saint Étienne à Bourges ${ }^{79}$. Malheureusement, on ne peut, encore une fois, que constater que les hagiographes se sont bien gardés de préciser ou de faire allusion aux sources qu'ils ont utilisées au cours de leur rédaction. Même l'auteur de la Vita B, dans son prologue, n'évoque pas la matière dont il aurait pu se servir pour la composition de son œuvre ${ }^{80}$. Il est du moins certain que ceux-ci ont trouvé chez Grégoire quelques clés de départ à l'extension du récit qui, probablement, n'est que pure imagination ${ }^{81}$.

Au terme de cette discussion autour de la forme des Vitae d'Ursin, on mesure à quel point il est difficile d'évaluer la valeur historique des informations transmises par la légende du saint. La tradition orale a du néanmoins beaucoup contribuer à la rédaction de ces deux Vies ${ }^{82}$. En 1925, le Bollandiste, H. Delehaye, prônait qu'on ne pouvait faire confiance qu'aux seules indications tirées du chapitre de l'Historia Francorum de Grégoire : toute anecdote ou tout renseignement supplémentaires devaient être consi-

77. DelEHAYE, Hyppolite, « De sancto Ursino, primo biturigensi episcopo », dans op. cit., p. 102.

78. Voir à ce sujet, LAUGARDIÈRE, Maurice de, op. cit., p. 22-24.

79. Sur cette question de l'invention hagiographique, voir GoulLET, Monique, op. cit., p. 214 : "Quand l'hagiographe ne sait rien de la biographie d'un saint, il invente en puisant dans le fonds commun des "bonnes actions" et des miracles de toutes sortes : il " invente ", c'est-à-dire qu'il " entre dans " (in-venit) quelque chose qui existait déjà, en se gardant surtout d'innover et de créer quelque suspense que ce soit ".

80. Pierre-André Sigal note que souvent les hagiographes font référence à leurs sources d'information, soucieux " de répondre à des critiques éventuelles ou à des accusations de mensonges ". Ils se justifient ainsi "face aux incrédules et aux détracteurs de miracles ", ce qui n'est pas le cas dans le prologue de la Vita B d'Ursin : SigAL, Pierre-André, "Le travail des hagiographes aux XI ${ }^{\mathrm{e}}$ et XII ${ }^{\mathrm{e}}$ siècles... ", dans op. cit., p. 151.

81. Dans son étude sur L'idéal de sainteté dans l'Aquitaine carolingienne d'après les sources hagiographiques (750-950) (op. cit., p. 21), Joseph-Claude Poulin identifie un type de vie de saints mérovingiens recomposé " par développement d'un passage de Grégoire de Tours ". Il donne le cas de la vie de saint Martial (BHL 5559-5560) s'appuyant sur les chapitres XXVII-XXVIII du Liber in Gloria Confessorum.

82. Sur la question de l'oralité cf. : Oldoni, Massimo, Culture del Medioevo, dotta, popolare, orale, Rome, 1999, surtout p. 78; DelehaYe, Hyppolite, Les légendes... op. cit., p. 6970; SigAL, Pierre-André, "Le travail des hagiographes aux XI et XII siècles... ", dans op. cit., p. 154. 
dérés de façon très critique, ce qui nous semble l'attitude la plus raisonnable à adopter envers ces textes ${ }^{83}$.

Enchaînant les lieux communs, les deux Vitae d'Ursin, mais encore plus la Vita B, sont de véritables combinaisons de citations bibliques ayant pour but, parallèlement à la transmission de la légende d'un saint, de présenter des modèles de vertu et de sainteté au peuple chrétien. Rédigées au cours du Moyen Âge central, elles demeurent, comme de nombreuses Vitae et autres pièces hagiographiques, des textes orphelins de leurs auteurs. À ce stade de la recherche, essayons de concevoir ce qui est à l'origine de leur rédaction.

\section{Hypothèses sur la rédaction}

À la suite de l'ensemble des observations faites sur le texte des Vitae, intéressons nous maintenant de plus près à ceux qui ont rédigé ces morceaux d'hagiographie et aux raisons qui ont motivé leur travail. Cette dernière partie de l'étude consacrée aux hypothèses de rédaction nous donnera également l'occasion de mener une réflexion sur la datation des textes.

On ne connaît le nom d'aucun des hagiographes à l'origine des Vitae A ou $\mathrm{B}$ de saint Ursin, ni du reste des épitomés. On ignore également où ces textes hagiographiques furent écrits, si c'est à Bourges même, ailleurs dans le Berry ou dans un autre lieu. Enfin, la date de la rédaction des deux plus importantes Vitae du saint demeure aussi inconnue même si, comme on l'a vu plus haut, les manuscrits conservés constituent en quelque sorte des limites chronologiques larges sur lesquelles on peut s'appuyer tout en restant prudent.

Essayons d'envisager la période à laquelle la première Vita, qu'il s'agisse de la version A ou de la B, de saint Ursin aurait pu être composée. Parmi les légendes sur les débuts du christianisme en Aquitaine, mais extérieures à Bourges, Ursin est désigné comme un disciple de saint Austremoine (BHL 845 et 848$)^{84}$ et de saint Martial ${ }^{85}$. Ceci met ainsi en

83. Ibidem, p. 151. C'était également l'avis de l'auteur du Patriarchum Bituricense au Xvi siècle (Le Patriarchum Bituricense est une histoire des archevêques de Bourges écrit par un bénédictin de Saint-Sulpice et publié d'après le manuscrit 233 de la Bibliothèque de Bourges : voir LABBE, Philippe, op. cit., p. 5-6 et 22).

84. Voir, sur cette question, l'ouvrage de MonNiER, Abbé S. M., Les saints d'Auvergne, histoire de tous les personnages de cette province honorés d'un culte public, Paris, 1899. Voir également l'étude du dossier hagiographique du saint par FoURNIER, Pierre-François, "Recherches sur l'histoire de l'Auvergne. Saint Austremoine, premier évêque de Clermont. Son épiscopat, ses reliques, ses légendes ", Bulletin historique et scientifique de l'Auvergne, 89, 1979, p. 417-471.

85. L'hagiographie de saint Martial, à notre connaissance, ne mentionne pas ce lien entre Ursin et l'évêque de Limoges. Néanmoins, l'historiographie a retenu cette idée qu'Ursin fut un compagnon de Martial. C'est le cas dans : BrimONT, Thierry de, Saint Ursin, son apostolat dans le Berry et son culte, Bourges, 1884, p. 24; DuCHESNE, Louis, op. cit., p. 9; CRISTIANI, Léon, "Liste chronologique des saints de France, des origines à l'avènement des Carolingiens ", dans R HE F, XXXI, 1945, p. 12-13; RÉAU, Louis, Iconographie de l'art chrétien, tome 3 : iconographie des saints, Paris, 1959, p. 1295. 
évidence les rivalités qui existaient dès le haut Moyen Âge entre les églises les plus importantes de cette grande région historique, chacune cherchant déjà à démontrer son antériorité sur la voisine ${ }^{86}$. Ainsi les hagiographes de saint Austremoine racontent qu'Ursin serait venu de Rome dans le cortège de l'apôtre d'Auvergne ${ }^{87}$, et ce n'est qu'ensuite, aidé du saint clermontois, qu'il aurait fondé l'Église de Bourges ${ }^{88}$. En effet, par ce moyen, les Auvergnats faisaient de l'Église de Bourges, dont les prétentions de primatie sur le Centre et même l'ensemble du Sud de la France étaient fortes, une église secondaire au regard de celle de Clermont. On comprend d'ailleurs que le clergé berruyer ait peu affectionné ces versions légendaires, l'archevêque de Bourges étant le métropolitain de celui de Clermont ${ }^{89}$. Néanmoins, la rivalité avec l'Église de Limoges, elle aussi sous l'autorité de la métropole berruyère, fut encore davantage poussée. En 1031, Aimon de Bourbon (1030-1070), archevêque de Bourges, convoquait deux conciles provinciaux dans le contexte de la Paix de Dieu : le premier dans sa cité épiscopale le $1^{\mathrm{er}}$ novembre et le second à Limoges, quelque trois semaines plus tard, le 18 novembre. Ce deuxième concile aurait permis d'asseoir, devant l'ensemble des évêques d'Aquitaine, l'apostolicité, revendiquée par le clergé local, du premier évêque de Limoges, saint Martial ${ }^{90}$. L'archevêque de Bourges y aurait déclaré que seul Martial était digne de porter le titre d'apôtre, que lui seul pouvait être nommé comme l'un des 72 disciples du Christ et donc, par déduction, que saint Ursin n'appartenait pas à ceux-ci. Afin d'imposer l'apostolicité du saint limougeaud, il aurait également rédigé et envoyé, à la suite du premier concile, un édit destiné aux évêques aquitains. Cependant, plusieurs études, réalisées au cours du $\mathrm{xx}^{\mathrm{e}}$ siècle, ont mon-

86. À ce propos, on consultera : EHLERS, Joachim, "Pilitik und Heiligenverehrung in Frankreich ", dans PETERSON, Jürgen (éd.), Politik und Heiligenverehrung im Hochmittelalter, (Vorkräge und Vorschungen, 42), Sigmarigen, p. 149-175.

87. Dans la Vita tertia (BHL 848) du saint, on peut lire : "Venerabilis igitur Dei cultor Austremonius partes Galliarum ingressus, et comitibus praefatis a se mutuo divisis, paucis tantum secum comitando, quos a beato Petro discipulos et socios accipere meruit, retentis, Necterium scilicet presbyterum, Ursinumque almificae probitatis virum, Mametum quoque habentem levitalis ordinis officium... " : Acta Sanctorum, Novembris, Tomus I, Bruxelles, 1887, p. 63.

88. "Itaque beatus Austremonius ad Bituricensium, incolas usque perveniens, populum adhuc in fide vacillantem suo studuit roborare documento et exemplo, et inibi aliquantisper commoratus, in ejusdem cathedrae pontificatu sublimavit beatum Ursinum, discipulum suum, nobilitatis apice pollentem et per omnia Deo placere studentem. " (Vita secunda, BHL 845) : Acta Sanctorum, Novembris, Tomus I, Bruxelles, 1887, p. 57. L'hagiographe de la Vita tertia reprend pratiquement intégralement ce passage à la Vita secunda.

89. DuCHESNE, Louis, op. cit., p. 123; voir aussi PÉRICARD, Jacques, Le diocèse de Bourges au haut Moyen Âge de saint Ursin à Audebert (IV siècle-1097). Essai sur le gouvernement épiscopal et les structures ecclésiastiques en Berry, thèse sous la dir. de Christian LAURANSONRosAz, Université Jean Moulin-Lyon III, 2004, p. 72, 278 et 284.

90. Ce concile fut entre autres la conséquence d'une revendication partie de l'abbaye Saint-Martial de Limoges à la suite, sans doute, de la rédaction de la légende aurélienne du saint. Pour les moines de Saint-Martial, le saint avait été un véritable apôtre et aurait reçu des mains du Christ lui-même sa mission. 
tré qu'une partie des actes des conciles de Bourges et de Limoges ${ }^{91}$, ainsi que l'édit d'Aimon sont des faux ${ }^{92}$ inventés par le célèbre moine de l'abbaye limougeaude, Adémar de Chabannes, défenseur acharné de la cause de l'apostolicité de Martial ${ }^{93}$. Cette manipulation, autant historique que politique, entraîna probablement le siège épiscopal de Bourges, " comptetenu de ses revendications provinciales, voire supra-provinciales, [...] à entrer dans la course ${ }^{94}$ " à l'apostolicité. Certains auteurs, comme Louis Réau, ont d'ailleurs émis une hypothèse très intéressante selon laquelle les textes ayant permis l'assimilation d'Ursin à Nathanaël, un des 72 disciples du Christ, " n'[auraient eu] pour but que d'“aligner" l'Église de Bourges sur les cathédrales des diocèses voisins auxquelles elle ne voulait pas être inférieure en ancienneté et de mettre saint Ursin sur le même plan que saint Martial de Limoges ${ }^{95}$ ".

Après ces mises au point, deux hypothèses s'offrent à nous quant à la date de rédaction des Vitae. On envisage aisément qu'en réaction au concile de 1031, l'Église de Bourges, se refusant à abandonner toute primatie à l'Église de Limoges ${ }^{96}$, ait commandé la rédaction d'une légende dans laquelle Ursin aurait été au moins l'égal de saint Martial. La première hypothèse consiste donc à penser que la Vita initiale de saint Ursin aurait pu être rédigée peu après 1031, ou tout au moins avant la fin du Xi ${ }^{\mathrm{e}}$ siècle. Il est alors fort possible qu'on en ait très rapidement fait une version amplifiée, la Vita B, comme les constatations mentionnées plus haut sur la forme et le fond du texte nous permettent de le supposer ${ }^{97}$.

91. Pour Bourges, voir HaRDouin, Jean, Acta conciliorum, Paris, 1714-1715, t. VI, pars 1, col. 847; pour Limoges, voir Migne, Jacques-Paul, P. L., t. CXLII, col. 1356-1400.

92. SALTET, Louis, «Les faux d'Adémar de Chabannes. Prétendues décisions sur saint Martial au concile de Bourges du $1^{\mathrm{er}}$ novembre 1031 ", Bulletin de littérature ecclésiastique, t. 27, 1926, p. 145-160; Callahan, Daniel F., "Adémar of Chabannes, Apocalypticism and the Peace of Council of Limoges of 1031 ", Revue bénédictine, t. 101, 1991, p. 32-49, surtout p. 35 ; BECQUET, Jacques, "Le concile de Limoges de 1031 ", Bulletin de la société archéologique et historique du Limousin, t. 128, 2000, p. 23-62. Pour un résumé de ces questions, voir Adémar de Chabannes, Chronique, (traduction par Y. Chauvin Yves et Plon, Georges), coll. « Miroir du Moyen Âge ", Turnhout, 2003, p. 13.

93. À propos d'A. de Chabannes, voir l'article de ARBELlot, François, «Étude historique sur l'ancienne vie de saint Martial ", dans Bulletin de la Société archéologique et historique du Limousin, LX, 1892, p. 213-269, celui déjà cité de Maurice COENS, "La " scriptura de sancto Fronto Nova " attribuée au chorévêque Gauzbert ", dans op. cit., p. 342-346, ou encore le livre de LANDES, Richard, PAUPERT, Catherine, Naissance d'apôtre. La vie de saint Martial de Limoges, un apocryphe de l'an Mil, Turnhout, 1991, mais surtout la thèse de Michel AuBrun, L'ancien diocèse de Limoges, des origines au milieu du XIe siècle, ClermontFerrand, Institut d'Etudes du Massif Central, 1981, p. 73-86, 204-206.

94. PÉRICARD, Jacques, op. cit., p. 284.

95. RÉAU, Louis, Iconographie de l'art chrétien, tome 3 : iconographie des saints, Paris, 1959, p. 1295.

96. Louis DuchesNe (op. cit., p. 19) écrivait que Bourges, à partir de la fin du Xle siècle, n'a plus jamais eu la prépondérance qu'elle souhaitait sur la province du Centre et même au-delà, raison qui poussa vraisemblablement encore davantage la rédaction d'une telle légende que celle d'Ursin.

97. Il semble plus logique que la vie de saint Ursin ait été augmentée plutôt que 
Cependant, s'appuyant sur des arguments majoritairement historiques, une seconde hypothèse se dégage de l'ensemble de cette étude : celle d'une rédaction de la Vita $\mathrm{B}$ en réaction immédiate au concile de Limoges, réécriture réalisée à partir de la Vita A, qui, elle, aurait préexisté à la date de $1031^{98}$. En effet, à la différence de la Vita A, Ursin est présenté dans la Vita B non pas comme l'un des 72 disciples du Christ mais comme le premier de ceux$\mathrm{ci}^{99}$. Du reste, l'hagiographe de la Vita B, en faisant d'Ursin le Nathanaël de l'Évangile, indication qui n'apparaît pas dans la Vita A, reléguait incontestablement saint Austremoine à l'arrière-plan et permettait au premier évêque de Bourges d'égaler la figure de Martial, dont la Vita prolixior (BHL 5552) en faisait le jeune cousin de Simon-Pierre et le fidèle compagnon de Jésus ${ }^{100}$. À la suite du concile de Limoges, le clergé berruyer considéra probablement que la Vita A était trop courte et commanda la rédaction d'une version plus longue et plus étoffée de la légende d'Ursin qui aurait davantage d'autorité. Même si l'hagiographe de la Vita B ne précise à aucun moment dans le prologue qu'il s'apprête à réécrire la vie de saint Ursin, il est difficile d'imaginer, dans l'hypothèse où la Vita A serait un résumé de la B, qu'on ait supprimé l'ensemble des éléments qui donnait un peu de gloire au premier évêque de Bourges. Ce dernier argument continue de nous convaincre que la Vita A précède la Vita B : la Vita B est donc une réécriture amplifiée de la Vita A, au sens que lui donne l'historienne, Monique Goullet ${ }^{101}$. Enfin, il n'est

condensée. Cependant, en 1951, le chanoine de LAUGARDIÈRE soutenait que la Vita A était un abrégé de la Vita B (op. cit., p. 21, note 2) ce qui sous-entendrait qu'un seul et même hagiographe serait à l'origine de la rédaction des deux textes. En suivant cette hypothèse, la Vita A correspondrait à une legenda liturgica, version résumée de la Vita B qu'on aurait réduit dans un but liturgique. Au contraire, Brigitte BEAUJARD écrit dans sa thèse que " plus le temps passait, plus la biographie d'un saint devenait détaillée " (op. cit., tome 1, p. 10). Monique Goullet note également qu'au XI ${ }^{\mathrm{e}}$ siècle, période de développement considérable de l'écrit, on atteint un point culminant dans la fréquence de la pratique de l'augmentation : GoULLET, Monique, "Une typologie des réécritures peut-elle éclairer la nature du discours hagiographique? ", dans Le riscritture agiografiche..., op. cit., p. 109-122 (surtout p. 115) et ead., op. cit., p. 246.

98. On a vu que le manuscrit le plus ancien conservé de la Vita A (Paris, BNF, ms. Lat. 13220) date des $\mathrm{X}^{\mathrm{e}}-\mathrm{XI}^{\mathrm{e}}$ siècles. Les folios transmettant la vie d'Ursin pourraient donc être antérieurs à 1031 mais l'observation du manuscrit n'a pas permis de dater précisément les folios.

99. "...adeo ut in numero septuaginta duorum discipulorum unus princeps post apostolos constitueretur ». (BHL 8413) : Acta Santorum, Novembris, Tomus IV, p. 109.

100. LANDES, Richard, PAUPERT, Catherine, op. cit., p. 9

101. L'amplification est à la fois une extension du texte originel par " un ajout de parties " et une expansion, c'est-à-dire " une dilatation stylistique de chacune des parties du texte "Souvent, les réécritures des vies de saints contiennent plus de citations bibliques, surtout évangéliques, utilisées pour développer des textes trop courts et pour mettre en évidence les similitudes de la vie du saint avec celle du Christ. Donc d'après la classification s'appuyant sur des notions d'intertextualité utilisée par Monique Goullet (calquée sur celle établie par Gérard GenETTE dans Palimpsestes. La littérature au second degré, Paris, 1982), la Vita A correspond à l'hypotexte et la Vita B à l'hypertexte. Le but d'une réécriture d'une vie de saint est d'" adapter l'hypertexte aux conditions historiques nouvelles, (de) le réorienter vers une finalité différente de celle de l'hypotexte ", GoulLET, Monique, "Vers une typologie des réécritures hagiographiques à partir de quelques 
pas nécessaire de revenir sur le fait que le début de la Vita B est identique à celui de la Légende aurélienne de saint Martial mais notons tout de même que, la Vie du saint limougeaud ayant été écrite entre 994 et $1028^{102}$, la rédaction de la Vita sancti Ursini B lui est postérieure ${ }^{103}$.

Par ailleurs, l'observation des folios 13 à 19 du plus ancien manuscrit conservant la Vita A (BNF, ms. Lat. 13220) apporte encore un nouvel élément en faveur de la seconde hypothèse. À un moment donné au cours des siècles, on a curieusement rayé, au folio 13 recto, le nom d'un des Sept évêques de la mission qui, après déduction, ne peut-être que celui de saint Martial de Limoges ${ }^{104}$. Compte-tenu de ce qu'on vient d'énoncer, il est frappant que ce soit justement le nom de l'évêque de Limoges qui ait été effacé, rature qui a sans doute été faite après 1029, date à laquelle les moines de Saint-Martial de Limoges « avancèrent que leur saint patron n'avait pas été

exemples du Nord-Est de la France ", dans Goullet, Monique, et HeInZELMAnN, Martin (dir.), op. cit., p. 109-144, plus spécialement p. 112), notamment en transformant le "statut " historique d'un saint. C'est exactement ce à quoi nous assistons entre la Vita A et la Vita B (voir ibidem, p. 111-112 et Goullet, Monique, op. cit., p. 111, 185-186). La Vita B d'Ursin n'est donc pas, comme l'indiquait Hyppolite DeLEHAYE ("De sancto Ursino, primo biturigensi episcopo ", dans op. cit., p. 108), la Vita prior du saint mais la Vita prolixior.

102. Bonnassié, Pierre, Sigal Pierre-André, Iogna-Prat Dominique, « La Gallia du Sud, 9301130 ", dans op. cit., p. 297.

103. Dans sa thèse sur L'idéal de sainteté dans l'Aquitaine carolingienne, Joseph-Claude Poulin (op. cit., p. 9-10) rattache, avec justesse, la Vita B de saint Ursin (BHL 8413) au cycle de la " légende aurélienne " attribué à un " pseudo-aurélien qui travaillait vers 955, et [à] plusieurs hagiographes aquitains à sa suite [qui] transformèrent leurs saints favoris en autant d'émissaires de saint Pierre ", similitude déjà soulignée précédemment. Selon lui, cette production aurait abouti à la " mise en circulation, dans la deuxième moitié du X ${ }^{\mathrm{e}}$ siècle, d'une série de fables " apostoliques " au profit de saint Martial d'abord (BHL 5552), puis d'Ausone d'Angoulême (BHL 828), Austriclinien et Valérie de Limoges (BHL 857 et 8476), Sylvain et Ursin de Berry (BHL 7723 et 8413) ". Cette datation de Joseph-Claude Poulin, qui place la rédaction de la Vita B au cours de la seconde moitié du $\mathrm{X}^{\mathrm{e}}$ siècle, ne nous semble pas justifiée au regard de l'ensemble des arguments énoncés plus haut, notamment celui du contexte historique, qui tendent à prouver que la Vita $\mathrm{B}$ a été écrite au $\mathrm{XI}^{\mathrm{e}}$ siècle.

104. " sanctus Dionysius Parisiacensis, sanctus Saturninus Tholosensis, Trophimus Arelatensis, Paulus Narbonensis... Austremonius Arvernensis, et sanctus Vatianus episcopus " (BHL 8412). Étienne-Michel FAILLON (op. cit., p. 419-422), cohérent avec sa datation des $\mathrm{V}^{\mathrm{e}}-\mathrm{Vl}^{\mathrm{e}}$ siècles de la Vita A de saint Ursin, en déduit, de son côté par contre, qu'à la suite du concile de Limoges, on dut adapter la liturgie de l'Église de Bourges et c'est ainsi que fut barré le nom de Martial sur le manuscrit puisqu'il avait été démontré au concile de Limoges que Martial avait précédé la mission des Sept. L'abbé C. CHEVALIER ("Les origines de l'Église de Tours ", dans op. cit., p. 479), réfutant cette thèse, précise d'ailleurs que le Sulpicien s'est bien gardé de mentionner qu'il y a en fait deux ratures sur le manuscrit de la Bibliothèque Nationale. En effet, au folio 13 recto, apparaissent deux ratures : la première est sans doute une erreur du copiste et on lit aisément, sous la rature, le nom de Trophime tandis que la seconde serait celle correspondant à l'évêque de Limoges. Toutefois, dans ce cas, il est pratiquement impossible de distinguer ce qui se cache sous le grattage. On peut seulement constater, en début de rature, la présence d'un " a " et, au milieu, la subsistance d'une haste qu'on peut imaginer être le reste d'un " l ". Enfin, on remarque que l'espace laissé est assez grand pour y voir l'inscription suivante «Martialis Lemovicensis". 
un simple confesseur [...] mais bien plus : un véritable apôtre ${ }^{105}$ ". Vraisemblablement, ce sont les moines de Saint-Martial de Limoges euxmêmes, à qui appartenait le manuscrit, qui grattèrent le nom de leur saint patron ${ }^{106}$. Il y a donc deux solutions à cette énigme : soit le libelle correspondant aux folios 13 à 19 du manuscrit Lat. 13220, dont on se souvient qu'il s'agit d'un recueil factice, n'a pas été rédigé à Saint-Martial de Limoges et donc, lors de son arrivée dans l'abbaye, à une date inconnue mais après 1029, il a été corrigé; soit le libelle provient de Saint-Martial de Limoges et a été composé avant 1029 puis corrigé par la suite, en quel cas la Vita A est antérieure à cette date. Étant donné l'importance du scriptorium de l'abbaye limougeaude, cette deuxième solution renforce notre conviction d'une rédaction de la Vita A d'Ursin en amont du concile de Limoges.

Des deux hypothèses envisagées, la seconde, qui place la rédaction de la Vita A avant les années 1930 du XI ${ }^{\mathrm{e}}$ siècle et celle de la Vita B à la suite du concile limougeaud, paraît à ce stade la plus convaincante, même si elle ne répond pas à toutes nos interrogations. En outre, si on persiste dans cette direction, l'absence pratiquement totale d'attention portée aux miracles du saint, constatée dans les deux Vitae, pourrait être un autre argument en faveur d'une datation vers le xe siècle de la Vita A. Les deux Vitae appartiendrait à ce courant hagiographique issu du haut Moyen Âge « réticen(t) vis-à-vis du " merveilleux » et du " miraculeux " ", attitude la plus répandue au $\mathrm{X}^{\mathrm{e}}$ siècle, selon Giulia Barone ${ }^{107}$.

L'étude des deux vies de saint Ursin, au-delà de l'analyse hagiographique, permet donc de vérifier encore une fois l'importance historique qu'eut cette quête des origines apostoliques dans laquelle se lancèrent les grandes provinces ecclésiastiques. À ce propos, I. Van’t Spijker faisait remarquer plus récemment que " [s]i les informations manquaient, par exemple dans les listes épiscopales, alors étaient créées de toutes pièces des Vies de saints totalement légendaires, dans lesquelles était mise en scène la continuité avec l'Église chrétienne primitive ou même apostolique. Ainsi l'Église locale recevait-elle une place à l'intérieur de la communauté universelle des croyants ${ }^{108} \ldots$.. ". Ce

105. LANDES, Richard, PAUPERT, Catherine, op. cit., p. 9.

106. «Les livres limousins eux-mêmes et spécialement [...] ceux de l'abbaye Saint-Martial [s]ans doute à de bien rares exceptions près, [avaient] été grattés pour les accommoder au système d'Adémar " (Duchesne L., op. cit., p. 115). R. LANDES mentionne également ce phénomène : "Dans le même temps, les moines limougeauds commencèrent à gratter et retoucher tous les passages susceptibles d'être opposés à leurs revendications nouvelles. Ils rachetèrent tout au long des $\mathrm{XII}^{\mathrm{e}}$ et XIII ${ }^{\mathrm{e}}$ siècles, les manuscrits liturgiques des abbayes voisines pour les corriger dans le même sens. Il résulta de cet effort soutenu un tel ensemble de preuves qu'en dépit d'une résistance opposée dès la première heure, ce culte apostolique de saint Martial né de l'initiative des moines de Limoges "fit loi en Limousin pendant plus de sept siècles" " (LANDES, Richard, PAUPERT, Catherine, op. cit., p. 9). 107. BARONE, Giulia, "Une hagiographie sans miracles. Observations en marge de quelques vies du $\mathrm{X}^{\mathrm{e}}$ siècle", dans Les fonctions des saints dans le monde occidental (III -XIII $^{e}$ siècle), Actes du colloque organisé par l'École française de Rome, Rome, 27-29 octobre 1988, Rome/Paris, 1991, p. 435-446.

108. VAN'T SPIJKER, Ienje, " Gallia du Nord et de l'Ouest. Les provinces ecclésiastiques de Tours, Rouen, Reims (950-1130) ", dans PhILIPPART, Guy (dir.), Hagiographies..., op. cit., 
commentaire semble parfaitement s'accorder avec le but qu'eut la rédaction d'une Vie d'Ursin dont il est difficile de douter que le commanditaire ne fut pas l'archiépiscopat berruyer ${ }^{109}$, à moins que la restaurationtion du chapitre Saint-Ursin en 1012 ait quelque rapport avec ce projet légendaire ${ }^{110}$.

L'hagiographie en Aquitaine traversa pendant le $\mathrm{IX}^{\mathrm{e}}$ siècle, période des invasions normandes, une période de stagnation pour connaître un regain de vitalité aux $\mathrm{X}^{\mathrm{e}}-\mathrm{XI}^{\mathrm{e}}$ siècles ${ }^{111}$. La renaissance au XI ${ }^{\mathrm{e}}$ siècle de l'église SaintUrsin transformée en collégiale, après un laps de temps pendant lequel sa présence disparaît de toute documentation, a très facilement pu encourager la rédaction d'une hagiographie sur le saint. Par une telle rédaction, on peut supposer qu'on ait voulu donner un nouvel essor au culte du saint dont les reliques, pendant cette période troublée, avaient été emmenées loin de Bourges ${ }^{112}$. Le chapitre de l'église y voyait également peut-être un moyen de s'affirmer dans le présent et d'encourager le pèlerinage vers la collégiale, véritable source de revenu pour l'établissement religieux, tout en renouant avec le passé prestigieux du sanctuaire ${ }^{113}$. Pierre-André Sigal faisait remarquer à son tour que « dans bien des cas, la coïncidence chronologique entre une tentative de réorganisation et de restauration et la confection d'une Vita [...] est frappante 114 ". Selon lui, il n'y a aucun doute sur le fait qu'un des objectifs d'une telle entreprise hagiographique était "d'ordre économique ": la notoriété d'un saint et encore plus la célébrité de son tombeau devenait un attrait indéniable aux yeux des pèlerins.

vol. 2, Turnhout, 1996, p. 239-290 et plus spécialement p. 243-244. Michel LAUWERS écrit, dans un article récent sur la " Mémoire des origines et idéologies monastiques. Saint-Pierredes-Fossés et Saint-Victor de Marseille au XI ${ }^{\mathrm{e}}$ siècle " (dans La mémoire des origines..., op. cit. Mélanges de l'EFR, tome 115, 2003, 1, p. 155-180), que " [1]es entreprises d'écriture [sont] souvent liées à des situations de crise ou à des moments de tension " (p. 179).

109. Pour Jacques PÉRICARD (op. cit., p. 285), l'origine de l'hagiographie d'Ursin pourrait se situer sous l'épiscopat de l'archevêque Gauzlin (1012-1030).

110. Après les importants dommages subis par l'église lors des invasions normandes pendant la seconde moitié du Ix ${ }^{\mathrm{e}}$ siècle, le culte aurait été rétabli dans l'église Saint-Ursin au début du XI $\mathrm{e}^{\mathrm{e}}$ siècle (cf. LE LuEL, Nathalie, Le portail Saint-Ursin de Bourges : recherches sur l'iconographie profane dans l'art religieux monumental de l'époque romane, doctorat en cours sous la direction de X. Barral i Altet, Université de Rennes 2). Une communauté de chanoines réguliers s'y installa, installation attestée par une charte de restauration datée de 1012, (Archives du Cher, 14 G 4 : Charte de restauration de Saint-Ursin par le vicomte de Bourges Gaufredus [Geoffroy le Noble] en 1012, reproduite dans une charte de Philippe $\mathrm{I}^{\text {er }}$ de 1102, présentée dans Prou, Michel, Recueil des actes de Philippe I ${ }^{\text {er }}$ roi de France [Paris, 1908], CXLVI, p. 368-372) et y prospéra tout au long du Moyen Âge.

111. Poulin, Joseph-Claude, op. cit., p. 11; Bonnassié, Pierre, Sigal Pierre-André, Iogna-PRAT Dominique, « La Gallia du Sud, 930-1130 », dans op. cit., p. 291-311.

112. OtTAWAY, John, « Bourges. ", dans BARRAl I Altet, Xavier (dir.), Le Paysage monumental de la France autour de l'an Mil, Paris, 1987, p. 262. Voir aussi Augonnet, Chanoine, Les reliques de saint Ursin, Bourges, 1894.

113. VAN'T SPIJKER, Ienje, « Gallia du Nord et de l'Ouest... », dans op. cit., p. 243. Sur la question de l'écriture ou de la réécriture par les communautés régulières de leur histoire, cf. Écrire son histoire. Les communautés régulières face à leur passé, actes du $5^{\mathrm{e}}$ colloque International du CERCOR, Saint-Étienne, 6-8 novembre 2002, Saint-Étienne, 2005.

114. SigaL Pierre-André, "Le travail des hagiographes aux XI et $\mathrm{XII}^{\mathrm{e}}$ siècles... ", dans op. cit., p. 150 . 
Quant à l'épitomé BHL 8415b, sa composition dut intervenir encore un peu plus tard, peut-être au cours du XII ${ }^{\mathrm{e}}$ siècle, puisque son auteur, à la différence des Vitae A et B, utilise le récit de la redécouverte du corps d'Ursin $\mathrm{au} \mathrm{VI}^{\mathrm{e}}$ siècle de Grégoire. Sans doute eut-elle pour dessein de rappeler encore une fois l'origine apostolique du fondateur de l'Église de Bourges?

Néanmoins, bien que nous ayons situé plus précisément la période de rédaction des Vitae d'Ursin, restent inconnus le lieu où elles furent composées ainsi que leurs auteurs. Il n'existe malheureusement que peu d'études sur la question de l'hagiographie à Bourges au Moyen Âge ${ }^{115}$. Remarquons que dans le prologue de la Vita B, l'hagiographe ne nous donne aucune indication sur sa propre personne, gardant, selon la tradition, volontairement l'anonymat ${ }^{116}$, ni sur le destinataire de l'œuvre ou encore sur les sources utilisées lors de la construction de la Vie. L'étude de la forme des textes a montré à quel point nos hagiographes étaient imprégnés des livres saints au point de ne plus citer, dans certains passages du texte, de manière littérale la Bible mais de mémoire. Cependant ces Vies furent-elles composées au sein d'un monastère ou au cour d'un chapitre cathédral? La rédaction eut-elle lieu à Bourges ou à l'extérieur de la cité berruyère? Serait-ce un clerc du chapitre cathédral de Bourges ou du chapitre de l'église Saint-Ursin qui aurait pu rédiger une telle vie ou un moine d'un des monastères de la ville?

Dans son étude, Joseph-Claude Poulin constate qu'aucune cellule religieuse en Aquitaine ne s'était spécialisée dans la production hagiographique, laissant ainsi entrevoir une géographie dispersée du genre littéraire ${ }^{117}$. Il atteste cependant d'une production à Bourges ${ }^{118}$. Notons que deux saints évêques de Bourges, saint Austrégésile (597-624) et saint Sulpice le Bon (624647), ont également bénéficié d'une hagiographie abondante dès la période mérovingienne. Les deux Vitae les plus anciennes des deux évêques, la Vita Austrigisili epicopi Biturigi (BHL 839) et la Vita sancti Sulpitii (BHL 7927-7928), seraient pratiquement contemporaines de leur épiscopat, datées du vII siècle, reprises plus tard au IX ${ }^{\mathrm{e}}$ siècle (respectivement BHL 841 et BHL 7930) ${ }^{119}$. Seule la première Vita de saint Austrégésile (BHL 839), d'après Joseph-Claude Poulin, serait l'œuvre d'un auteur étranger à l'Aquitaine ${ }^{120}$. Il n'y aurait donc aucune objection à ce que les Vitae d'Ursin aient été écrites dans la région mais on ne peut cependant pas affirmer que cela fut à Bourges ${ }^{121}$.

115. On pourra toutefois se reporter à l'ouvrage collectif dirigé par HEINZELMANN, Martin (dir.), Manuscrits hagiographiques et travail des hagiographes, (Francia, band 24), Sigmaringen, 1992.

116. SIGAL, Pierre-André, "Le travail des hagiographes aux XI et XII siècles... ", dans op. cit., p. 171.

117. Pouln, Joseph-Claude, op. cit., p. 37.

118. Ibidem.

119. Ibid., p. 16 et 168-177. Selon Louis DucheSNE (op. cit., p. 25-26), les deux Vitae mérovingiennes seraient de main contemporaine.

120. Poulin, Joseph-Claude, op. cit., p. 177.

121. SigAL, Pierre-André, "Le travail des hagiographes aux Xle et XII siècles... ", dans op. cit., p. 171 : Pierre-André Sigal constate que "dans la très grande majorité des cas, l'auteur fait partie de la communauté locale». 
Rédigées dans une volonté d'édification du saint, les Vitae du premier évêque de Bourges, comme on l'a déjà esquissé, apportent moins sur sa personnalité, dont d'ailleurs on devait connaître peu de chose, que sur l'idéal de sainteté en lui-même ${ }^{122}$. En effet, on peut supposer que la première Vita d'Ursin fut composée aussi tardivement à cause du manque de connaissances sur l'épiscopat du fameux évêque. C'est sans doute la raison pour laquelle les hagiographes d'Ursin empruntèrent des passages à d'autres vies de saints et, dans le cas de la Vita $\mathrm{B}$, un schéma de composition très proche de la Vita prolixior de saint Martial (BHL 5552). Comme l'écrivait très justement Jean Leclercq en 1963, " les Vies de saints sont, pour une grande part, l'illustration, au moyen d'exemples pratiques, des leçons que tout chrétien doit puiser dans la Bible ${ }^{123}$ ". En effet, chaque épisode de la vie de saint Ursin est l'occasion d'un parallèle avec la vie du Christ elle-même ${ }^{124}$. Ainsi l'hagiographe se sert de l'Écriture Sainte pour donner à son texte un crédit incontestable : la Bible est en quelque sorte le garant des faits qu'il énonce mais la Vita est, à son tour, un commentaire biblique adressé aux fidèles ${ }^{125}$.

On peut aussi se demander quelle était la destination de ces Vitae : étaient-elles réservées à la lecture lors d'exercices conventuels (repas) ou lors de l'office, ou avaient-elles encore pour but la prédication aux fidèles ${ }^{126}$ ? On peut du moins être certain qu'un des principaux buts de rédaction de ces deux textes hagiographiques était la célébration liturgique de la fête de saint Ursin, chacune des vies étant découpée en 9 leçons ${ }^{127}$.

Au terme de cette étude, ressortent plusieurs facteurs qui semblent avoir été à l'origine de la rédaction de la majorité du corpus hagiographique dédié au premier évêque de Bourges, Ursin, à l'exception de l'œuvre de Grégoire de Tours. La quête de l'archiépiscopat berruyer pour prouver l'ori-

122. Delehaye, Hyppolite, Les légendes..., op. cit., p. 23; Voir au sujet de l'idéal de sainteté, Poulin, Joseph-Claude, op. cit., p. 99 sqq.

123. LECLERCQ, Jean, "L'écriture sainte dans l'hagiographie monastique... ”, dans op. cit., p. 110 .

124. En rapport avec cette observation, Jean LECLERCQ ("L'écriture sainte dans l'hagiographie monastique...", dans $o p$. cit., p. 115 et 116) notait que "le thème de l'imitation de Jésus Christ est, de tous, le plus fréquent " et que " toute action doit s'appuyer sur l'auctoritas de la Bible, [qu'] elle doit avoir un précédent dans un texte qui constitue un modèle et sa garantie ". Plus récemment, Monique Goullet (op. cit., p. 210) écrivait : le "modèle de tout récit hagiographique, c'est la vie du Christ, de sa naissance à sa Passion pour ce qui est de la vie terrestre, et surtout sa résurrection ".

125. LECLERCQ, Jean, "L'écriture sainte dans l'hagiographie monastique... ", dans op. cit., p. 117.

126. MERDRIGNAC, Bernard, Les Vies de saints bretons durant le haut Moyen Âge, la culture, les croyances en Bretagne (VII -XII siècles), Rennes, 1993, p. 11-13. Pour Pierre-André SigAL, dans son article sur "Le travail des hagiographes aux XI ${ }^{\mathrm{e}}$ et XII ${ }^{\mathrm{e}}$ siècles... ", dans op. cit., p. 150, le premier but d'une Vie de saint était liturgique "destin[ée] avant tout à la célébration de la fête du saint et, secondairement, à des lectures pieuses ", la seconde fin étant "d'ordre économique" comme écrit plus haut.

127. Aigrain, René, op. cit., p. 166; SigAL Pierre-André, L'homme et le miracle dans la France médiévale (XI'-XII siècle), Paris, 1985, p. 10 ; DuBors Dom Jacques, Lemâ̂TRE Jean-Loup, Sources et méthodes de l'hagiographie médiévale, Paris, 1993, p. 89-98. 
gine apostolique du siège, de manière similaire à de nombreux clergés contemporains, est indubitablement le premier de ces facteurs, quête qui s'est faite dans un but de prestige, mais également dans l'espoir de s'affirmer face, essentiellement, aux prétentions limougeaudes. Deuxième facteur déterminant dans la création d'un tel ensemble hagiographique, la fondation du chapitre Saint-Ursin, en 1012, a vraisemblablement aussi motivé la constitution d'une légende. Rédigée vers la fin du $\mathrm{X}^{\mathrm{e}}$ siècle ou le début du $\mathrm{XI}^{\mathrm{e}}$ siècle, l'hagiographie connaissait une nouvelle floraison, la Vita A de saint Ursin aurait très vite semblé trop insuffisante et, les événements se précipitant autour de la métropole berruyère, une Vita $\mathrm{B}$, plus longue, amplifiée, aurait vu le jour, peu après 1031. 


\section{RÉSUMÉ}

La légende de saint Ursin raconte qu'il aurait été le premier évêque de Bourges et le grand évangélisateur du Berry. Son histoire est donc directement associée à celle des origines de l'Église berruyère. Le présent article examine, pour la première fois, les deux Vitae (BHL 8412 et BHL 8413) qui composent le coeur du corpus hagiographique d'Ursin, avec pour but de déterminer la date, le lieu et principalement les motifs de leur rédaction. L'observation attentive du couple de textes, construits suivant une structure fort proche et selon des procédés hagiographiques classiques, met en évidence une composition quasi-simultanée des deux Vies d'Ursin dans le contexte du mouvement historique de quête des origines apostoliques au $\mathrm{XI}^{\mathrm{e}}$ siècle. Motivée par la détermination du clergé berruyer à prouver l'ancienneté de la fondation du siège épiscopal, notamment en réaction aux fortes revendications auvergnates et surtout limougeaudes, la rédaction d'une première Vie (BHL 8412), entre la fin du $\mathrm{X}^{\mathrm{e}}$ siècle et le début du $\mathrm{XI}^{\mathrm{e}}$ siècle, fut très rapidement suivie d'une réécriture amplifiée, correspondant à la seconde Vita d'Ursin (BHL 8413).

\section{ABSTRACT}

The legend of Saint Ursin relates that he would have been the first bishop of Bourges and the great evangelist of the Berry area. His history is therefore directly linked to that which tells the origins of Bourges' Church. The present article examines, for the first time, the two Vitae (BHL 8412 et BHL 8413) which composed the heart of Ursin's hagiographic corpus, with the aim to determine the date, the place and mainly the reasons for their redaction. The careful observation of the two texts, constructed on the basis of a really similar structure and according to classic hagiographic processes, reveals an almost simultaneous composition of Ursin's two Lives in the context of the historical movement of quest for apostolic origins during the $11^{\text {th }}$ century. Motivated by the determination of Bourges' clergy to prove the old age of the episcopal see's foundation, notably in reaction to strong claims coming from Auvergne and, especially, from Limoges, the redaction of a first Life (BHL 8412), between the end of the 10 th century and the beginning of the $11^{\text {th }}$ century, was very quickly followed by an expanded rewritten version, corresponding to the Ursin's second Vita (BHL 8413). 